Review

\title{
Phase diagrams of lipid mixtures relevant to the study of membrane rafts
}

\author{
Félix M. Goñi ${ }^{\text {a,* }}$, Alicia Alonso ${ }^{\text {a }}$, Luis A. Bagatolli ${ }^{\text {, }}$, Rhoderick E. Brown ${ }^{c}$, Derek Marsh ${ }^{\text {d, }}$ \\ Manuel Prieto ${ }^{\mathrm{e}}$, Jenifer L. Thewalt ${ }^{\mathrm{f}, \mathrm{g}}$ \\ a Unidad de Biofísica (Centro Mixto CSIC-UPV/EHU), and Departamento de Bioquímica, Universidad del País Vasco, P.O. Box 644, 48080 Bilbao, Spain \\ ${ }^{\mathrm{b}}$ MEMPHYS, Center for Biomembrane Physics, Dept. of Biochemistry and Molecular Biology, University of Southern Denmark, Odense, Denmark \\ c The Hormel Institute, University of Minnesota, 801 16th Ave. NE, Austin, MN 55912, USA \\ ${ }^{d}$ Max-Planck-Institut für biophysikalische Chemie, Abteilung Spektroskopie, 37070 Göttingen, Germany \\ e Centro Química-Física Molecular, IST, Av. Rovisco Pais, 1049-001, Lisbon, Portugal \\ ${ }^{\mathrm{f}}$ Dept. of Molecular Biology and Biochemistry, Simon Fraser University, 8888 University Drive, Burnaby, BC, Canada V5A 1S6 \\ ${ }^{g}$ Dept. of Physics, Simon Fraser University, 8888 University Drive, Burnaby, BC, Canada V5A 1 S6
}

\section{A R T I C L E I N F O}

\section{Article history:}

Received 21 April 2008

Received in revised form 10 August 2008

Accepted 9 September 2008

Available online 7 October 2008

\section{Keywords:}

Membrane rafts

Lipid phase diagrams

Phosphatidylcholine

Sphingomyelin

Cholesterol

\begin{abstract}
A B S T R A C T
The present paper reviews the phase properties of phosphatidylcholine-sphingomyelin-cholesterol mixtures, that are often used as models for membrane "raft" microdomains. The available data based on $\mathrm{X}$-ray, microscopic and spectroscopic observations, surface pressure and calorimetric measurements, and detergent solubilization assays, are critically evaluated and rationalized in terms of triangular phase diagrams. The remaining uncertainties are discussed specifically and separately from the data on which a consensus appears to exist.
\end{abstract}

(c) 2008 Elsevier B.V. All rights reserved.

\section{Introduction}

For many years, lipids were considered to be the randomly organized building blocks of biological membranes, with the integral proteins floating in a two-dimensional lipid 'sea' and freely diffusing laterally in the lipid matrix unless their movements were restricted by associations with other cellular components. Early evidence that lipids could laterally segregate in membranes under certain conditions, and could form distinct lipid domains with particular structural

Abbreviations: AFM, atomic force microscopy; BSM, brain sphingomyelin; Chol, cholesterol; DMPC, dimyristoylphosphatidylcholine; DOPC, dioleoylphosphatidylcholine; DPH, diphenyhexatriene; DPPC, dipalmitoylphosphatidylcholine; DPPE, dipalmitoylphosphatidylethanolamine; DSC, differential scanning calorimetry; ESR, electron spin resonance; FLIM, fluorescence lifetime imaging; FM, fluorescence microscopy; FRET, fluorescence resonance energy transfer; GUV, giant unilamellar vesicle; $H_{I}$, inverted hexagonal phase; $K_{\mathrm{p}}$, partition coefficient of a given probe between two lipid phases; $\mathrm{L}_{\alpha}$, fluid, or liquid crystalline, lamellar phase; $\mathrm{L}_{\beta}$, gel, or solid-ordered, lamellar phase; $\mathrm{L}_{\mathrm{d}}$, liquid-disordered, or fluid-disordered, lamellar phase; $\mathrm{L}_{\mathrm{o}}$, liquid-ordered, or fluid-ordered lamellar phase; LUV, large unilamellar vesicles; MS, mass spectrometry; NMR, nuclear magnetic resonance; $\mathrm{P}_{\beta^{\prime}}$, rippled lamellar phase; $\mathrm{PC}$, phosphatidylcholine; PFG, pulsed field gradient; POPC, 1-palmitoyl-2-oleoylphosphatidylcholine; PSM, palmitoylsphingomyelin; SM, sphingomyelin; $T_{\mathrm{m}}$, mid-point transition temperature of a phospholipid gel-fluid thermotropic transition

* Corresponding author. Fax: +34946013360

E-mail address: felix.goni@ehu.es (F.M. Goñi). characteristics (i.e. a particular lipid phase) was reported in the early 1970s by Phillips et al. [1], who evaluated lateral mixing of different phosphatidylcholine (PC) species using differential scanning calorimetry (DSC), by Shimshick and McConnell [2], who mapped out phase diagrams for lateral phase separation by using electron spin resonance (ESR), by Grant et al. [3], who visualized lipid domains by freezefracture electron microscopy, and by Lentz et al. [4], who used fluorescence anisotropy to show nonideal mixing among phosphatidylcholines containing saturated and unsaturated chains. Detailed nuclear magnetic resonance (NMR) studies of sphingomyelin in bilayers by Schmidt et al. [5] prompted the suggestion that this sphingolipid might form microdomains in biological membranes. Gebhardt et al. [6] considered the known lipid compositional heterogeneity in biological membranes and predicted that lipid lateral segregation might occur under particular environmental conditions such as those that mimic a physiological state.

At nearly the same time, Jain and White [7] proposed a 'plate model' of membrane structure. The main tenet of this model was: 'The separation of ordered regions from the disordered regions (fluid) is a natural consequence of specific intermolecular interaction and lattice deformation,' and the authors suggested that considering data from the viewpoint of this scenario might explain and unite the accumulated experimental evidence from many different laboratories under a single hypothetical structure. Many of the ideas and observations related to 
the non-random lateral mixing of membrane lipids as related to the possible existence of microdomains were discussed in early comprehensive reviews by Thompson and colleagues $[8,9]$. Later, Ipsen et al. [10] introduced the distinction between $\mathrm{L}_{\mathrm{d}}$, or fluid-disordered, and $\mathrm{L}_{\mathrm{o}}$, or fluid-ordered lamellar phases. The latter would combine free rotational and translational diffusion of the lipid molecules (as found in the $\mathrm{L}_{\alpha}$ liquid crystalline phases) with a low proportion of gauche rotamers in the hydrocarbon chains (i.e. high order rather than low order), as is usually found in the $\mathrm{L}_{\beta}$ or gel phases. These ideas did not receive much attention, particularly from biologists, until the 'raft' hypothesis was postulated by Simons and Ikonen in 1997 [11]. This hypothesis suggested the existence of lipid rafts, transient microdomains enriched in sphingolipids and cholesterol that would be associated with specific proteins involved in cellular functions such as intracellular lipid traffic and cell signalling. The seminal 1997 paper has originated literally thousands of projects and publications in multiple areas of cell biology, biochemistry and biophysics.

A number of lipid mixtures have been used to mimic the biophysical characteristics of rafts in membrane model systems; recent studies have confirmed the importance of using natural raft mixtures rich in SM, Chol and small amounts of glycosphingolipids, such as gangliosides, compared to mixtures containing "model" raft lipids such as DPPC [12-14]. Therefore, the experimental mixtures designed to mimic putative coexisting raft and non-raft domains are usually composed of ternary mixtures of unsaturated phospholipids (DOPC or POPC), SM (synthetic [16:0] or natural), Chol, and in some cases small amounts of the ganglioside $\mathrm{G}_{\mathrm{M} 1}$, with all components present in the mix at specific molar fractions. Substantial efforts have been made in different laboratories to fully characterize the phase diagrams of what might be termed the canonical raft mixture. This review will focus on a number of aspects involved in characterizing lipid phase diagrams, such as the presence of micron-sized vs. nanometre-sized domains in the bilayers used for experimentation, and the various strategies for accurately assigning tie-lines for phase diagrams, and will also consider some of the still unsolved aspects of the physical behaviour of these deceptively simple-looking lipid mixtures. It will be important, along our discussion, to keep in mind the conceptual difference between "phases", thermodynamic idealizations defined under equilibrium conditions, characterized by a set of physical parameters, and "domains", real objects with the physical properties of a given phase. Needless to say, studies on phases and phase behaviour of lipids are meaningless, if not misleading, when equilibrium conditions are not attained prior to the measurements.

\section{Phase diagrams and domain sizes from fluorescence spectroscopy}

\subsection{Phase diagrams}

In this section, phases in the presence of cholesterol will be described in the framework of the conceptual distinction between $\mathrm{L}_{\mathrm{o}}$ and $L_{d}$ phases. It should be stressed that a lively discussion exists about these concepts, and interesting results (e.g., [15], using X-ray diffraction, and [16], using NMR data), cast doubts on their separate existence for the "canonical" binary DPPC/Chol mixture. The starting point in the characterization of lipid phases/domains is the construction of the lipid phase diagram. Lipid phase diagrams can be derived from thermodynamic data obtained by techniques such as differential scanning calorimetry (DSC), or from spectroscopic approaches such as NMR, ESR, or fluorescence, as described below. Fluorescence and ESR involve the use of probes, but this is not an obstacle to acquiring relevant data, since the high sensitivity of these techniques allows for the system to be only minimally perturbed. The probe:lipid ratio can be as low as 1:1000, so that in thermodynamic terms, the probe may be seen as an ideally diluted solute. The probe distribution between two phases (designated 1 and 2) is a very important property, and is determined from its partition coefficient $K_{\mathrm{p}}$ (Eq. (1)) as,

$K_{\mathrm{p}}=\frac{n_{2} / X_{2}}{n_{1} / X_{1}}$

where $n_{1}$ and $n_{2}$ are the number of moles of probe in phase 1 and 2 , respectively, and $X_{\mathrm{i}}$ the mole fraction of phase $i$. Phases 1 and 2 are any two co-existing lipid phases, usually either gel and fluid, or both fluid as in $\mathrm{L}_{\mathrm{o}}$ and $\mathrm{L}_{\mathrm{d}}$. $K_{\mathrm{p}}$ is meaningful only when a tie-line (see below) is considered. The primary requirement for obtaining $K_{\mathrm{p}}$ is that the parameter under study must vary significantly from one phase to another, and it is important to note that any parameter fulfilling this requirement may be used [17].

The determination of phase boundaries is the first step in the derivation of the phase diagram, which provides a means of viewing the relationships between compositions and phase changes. A graphical representation of the dependence of a parameter on lipid composition may show an abrupt change in the slope of the plot once a phase boundary is crossed. It should be stressed that the ability to detect a potential phase change is dependent on the ability of the probe to successfully incorporate into both phases, while exhibiting a change in an observable property in parallel with the phase transition. Technical problems resulting in inadequate or non-existent probe incorporation can lead to missing the presence of a new phase, thus the lack of evidence by one technique, does not rule out the existence of a new phase. An example of the derivation of a ternary phase diagram of a raft model system in which phase analysis required several different fluorescence methodologies is shown in Fig. 1 [18]. DPH anisotropy was a suitable reporter for collection of the data shown on the left-hand side of the diagram (PC/Chol region), while the use of methods based on DPH fluorescence quenching by spinprobes was necessary to obtain the data shown on the right-hand side of the diagram (PSM/Chol region). An additional complication in data collection came from the observation that the phase boundaries inside the Gibbs-triangle (i.e. mixtures of three lipids), could not be accurately determined at a higher temperature $\left(37^{\circ} \mathrm{C}\right)$ even though they were evident in binary systems at $37{ }^{\circ} \mathrm{C}$. This example illustrates why several methodologies should be explored in the collection of phase diagram data: Multiple methods may often be necessary both to validate results and also to help ensure that no phase change data is missed because of the technological limitations of any given method.

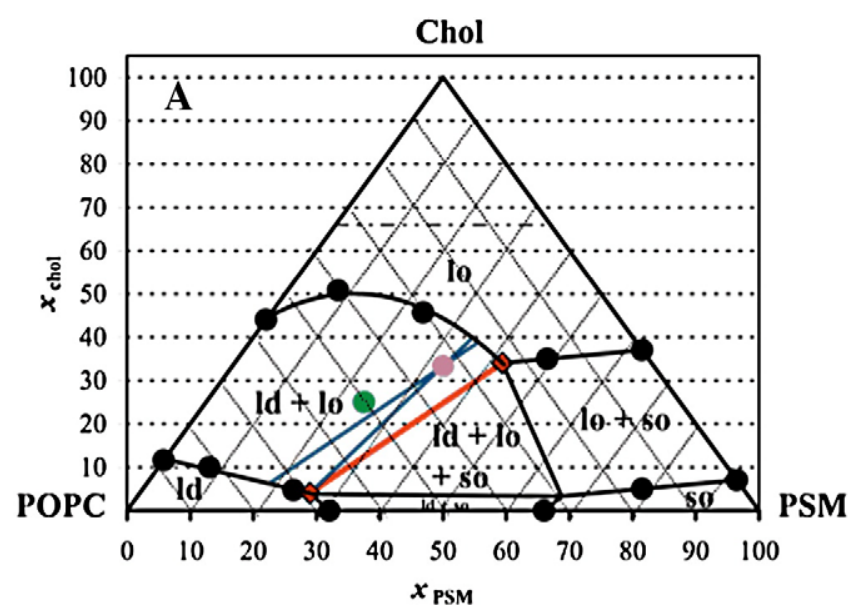

Fig. 1. $\mathrm{PSM} / \mathrm{POPC} / \mathrm{Chol}$ phase diagram at $23{ }^{\circ} \mathrm{C}$. The circles represent experimental points. The red (quasi) tie-line on the tie-triangle describes the $\mathrm{L}_{\mathrm{o}} / \mathrm{L}_{d}$ composition at the right of which there is also gel phase. The blue tie-lines limit the region for the possible tie-lines that contain the 1:1:1 composition. The purple point marks the locus of 1:1:1 composition, and the green point marks the locus of 2:1:1 composition. The dashed horizontal line for $x_{\text {chol }}=0.66$ represents the cholesterol solubility limit in the lipid bilayer. Figure derived from de Almeida et al. [186]. 
In this stage of the analysis, thermodynamic consistency should be checked, as this not only enables correction of errors, but also assists in the detection of hidden phases. For instance, in the data portrayed in Fig. 1, thermodynamic rules dictate the existence of the inner triangle (tie-triangle) since the orientation of the phase boundaries imply three-phase coexistence. Experimental evidence for this three-phase coexistence region was obtained by fluorescence lifetime imaging (FLIM) in the related system DOPC/DPPC/Chol by de Almeida et al. [18]. Inside a phase coexistence region, the relevant tie-lines must be defined. Along a tie-line the relative amounts, but not the composition, of the phases vary according to the lever rule. The following example illustrates the lever rule application: We may assume that one of the two lines crossing the 1:1:1 lipid composition point (i.e. the purple point in the diagram shown in Fig. 1) is a tie-line (this issue will be discussed later). Upon lipid mixing, the system separates into $\mathrm{L}_{0}$ and $L_{d}$ phases. The amount of $L_{o}$ phase (the majority phase, in this case) is represented by the distance from the $1: 1: 1$ point to the $L_{d}$ boundary divided by the total tie-line length, and the reverse applies to the determination of the amount of the $L_{d}$ phase (see Section 8 for more details). The composition of each phase is constant along the tieline, and is indicated by the intersection of the blue line with the phase boundaries. It should be stressed that some experiments must be carried out at compositions along the tie-line, because if the phase composition changes, the partition coefficient can no longer be defined, and some methodologies such as the quantitative fluorescence resonance energy transfer (FRET) formalisms that are used in the determination of domain sizes, cannot be applied. The tie-lines should be experimentally determined, and (contrary to common belief) they do not necessarily converge on a single point out of the diagram. The determination of tie-lines is a complex and timeconsuming procedure that is based on the systematic determination of the relative phase amounts in the region of interest. Such a determination can be carried out using any suitable reporter probe/ methodology, and global analysis according to the lever rule may be applied.

A detailed example of the application of this methodology will be presented in Section 8. An alternative method could entail analyzing the phase compositions (which should be invariant along a tie-line) and then applying an identical methodological analysis. Detergent extraction should not be used in the determination of tie-lines [19]. Kraft et al. [20] have used an approach involving high-resolution secondary ion mass spectrometry (MS), with lateral resolution allowing the determination of lipid composition of $100 \mathrm{~nm}$ domains in supported membranes.

However, in most situations, the tie-line can actually be estimated solely from thermodynamic rules with only a small degree of uncertainty, thus avoiding the need for experimental analytical work. Among those rules the restriction that two tie-lines may not cross inside the diagram (fan-wise orientation) is very useful. It is therefore possible to draw (with reference to the diagram shown in Fig. 1) two lines that are, respectively, parallel to the side of the triangle or to the red line, since these two lines are also tie-lines. However, because the line parallel to the left side of the triangle would cross the red line, the uncertainty in this case is even lower, as we are confined to the two blue lines. This limitation determines the maximum uncertainty of the tie-line slope (i.e. the tie-line is in between the two blue lines), and this approximation is a reasonable one for most purposes. As described, the tie-line orientation in this specific case is solely based on thermodynamic considerations, and not "estimated from work by others on different mixtures" as claimed by Zhao et al. [21]. In phase diagrams, the phase co-existence regions are clearly defined, and according to the established definition of rafts ( $\mathrm{L}_{\mathrm{o}}$ domains dispersed in the $\mathrm{L}_{\mathrm{d}}$ phase), rafts could exist for the lipid compositions shown at the left-hand side of the diagram in Fig. 1. Phase diagrams also allow for the reconciliation of a substantial body of literature data that had shown apparent discrepancies in results. Considerations about phase diagrams as the one shown in Fig. 1, and similar examples for other systems may be found in the literature (for reviews see [22-24]).

\subsection{Raft sizes}

The next step after the construction of a phase diagram is the determination of domain sizes. FRET has been used successfully to this aim. Because the relevant formalisms have already been described elsewhere [25], we will provide only a brief outline of the quantitative procedure here. At one extreme, in cases involving very large domains, FRET is much less efficient for a donor and acceptor FRET pair that tend to localize to different phases, resulting in the so-called infinite phase separation limit relative to the Förster radius, $\mathrm{R}_{\mathrm{o}}$. On the other hand, if the domains are very small, no decrease in efficiency is observed-the probe distribution is nearly random, as if only one phase was present, rather than two.

In between these two extreme limits are the biologically relevant domains, which tend to range from 10 to $100 \mathrm{~nm}$ in size, although there are also exceptions, in that some micrometer-sized domains do exist in biologically significant systems (see Section 3). In the case of the intermediate-size domains, boundary effects are relevant: donors in one phase can transfer to acceptors in the same phase but can also interact with acceptors located in a separate phase. This results in an experimental efficiency that is higher than that of a case of 'infinite phase separation,' but lower than the efficiency that would be associated with a single homogeneous phase in which probes would be expected to distribute randomly. A more sophisticated approach utilizes the global analysis of donor fluorescence decay in the absence and presence of acceptor. This approach takes advantage of the sensitivity of FRET to the local acceptor concentration in order to allow the determination of the apparent partition coefficients of the probes. Comparison of these values with the partition coefficients obtained by other, size-independent methodologies, provides information on the size of the domains at the nanoscale.

This approach is exemplified by the determination of the sizes of different regions in the ternary diagram described in Fig. 1 [26]. The study was carried out along the tie-line crossing the 1:1:1 composition point, and the probes used as the FRET pair were NBD-DPPE and Rhodamine-DOPE, which tend to localize in the $\mathrm{L}_{o}$ phase and in the $\mathrm{L}_{\mathrm{d}}$ phase, respectively. As described previously, this tie-line was considered to be in between the two blue lines shown in Fig 1. Fig. 2 shows a plot of the FRET efficiency vs. the molar fraction of the $\mathrm{L}_{0}$

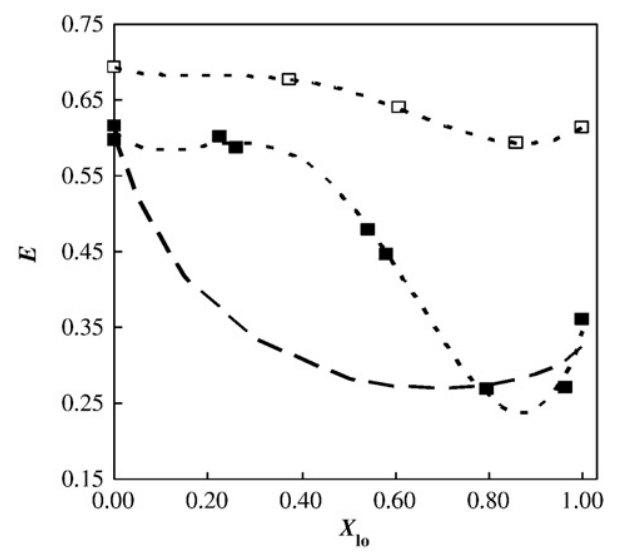

Fig. 2. Variation in FRET efficiency as a function of $\mathrm{L}_{\mathrm{o}}$ phase mole fraction between the donor/acceptor pair NBD-DPPE/Rhodamine-DOPE in PSM/POPC/Chol large unilamellar vesicles. Data is shown along the tie-line containing the lipid mixture 1:1:1 (mol:mol: $\mathrm{mol}$ ) at $23{ }^{\circ} \mathrm{C}$ with $0.2 \mathrm{~mol} \%$ of acceptor ( $\boldsymbol{\square}$ ); for comparison, data for the binary mixture DMPC/Chol along the tie-line at $30{ }^{\circ} \mathrm{C}$ is also shown ( $\square$ ) [28]. The theoretical line for infinite phase separation for large domains for the ternary mixture is also shown (thick broken line). See text for details. Adapted from de Almeida et al. [26]. 
phase. In the absence of domains $\left(X_{\mathrm{Lo}}=0\right)$, the experimental efficiency is, as expected, very close to the theoretical value, which is based on the assumption that the probes are randomly distributed. Up to a value of $X_{\mathrm{Lo}}=0.3$, there is no significant decrease of FRET efficiency, which indicates that the domains are very small (less than $20 \mathrm{~nm}$ ) as compared to $R_{0}$. Although the presence of such small domains is not detected by FRET, their existence is certain because they are, by definition of the plot area, in a phase-coexistence region. In agreement with this study, small domains were also reported on the same compositional region of a similar system (BSM was used, instead of PSM) by Frazier et al. [27]. Beyond the $X_{\mathrm{Lo}}=0.3$ limit, the efficiency of FRET decreases because the domains become larger, and therefore donor and acceptor probes are farther apart and are less likely to interact. It follows that if only large domains were present, the FRET efficiency curve would be parabolic (as is the thick broken line in Fig. 2). The actual experimental curve is much more complex, indicating that there are likely alterations in the size of the domains along the tie-line. In fact, in the case of very low $\mathrm{L}_{\mathrm{o}}$ fractions, the $\mathrm{L}_{\mathrm{o}}$ domains are quite small (less than $20 \mathrm{~nm}$ ), and enlarge with increasing $\mathrm{L}_{\mathrm{o}}$ mole fraction, suggesting that a nucleation process is occurring and that nucleation results in the generation of the larger domains. For very large $\mathrm{L}_{\mathrm{o}}$ fractions, the experimental FRET efficiency value approaches a value that corresponds to the idealized case of infinite domains, which means that the distance between the FRET pair is greater than $\sim 75 \mathrm{~nm}$. An advantage of representing FRET efficiency as a function of the $L_{o}$ mole fraction is that different systems characterized by $\mathrm{L}_{\mathrm{o}} / \mathrm{L}_{\mathrm{d}}$ phase coexistence can be compared in a single plot. Fig. 2 also shows the FRET efficiency for the binary DMPC/Chol system at $30{ }^{\circ} \mathrm{C}[28]$, and it is apparent that in this binary system the decrease in FRET is much less pronounced than in the ternary system. This finding indicates that the size of the domains in binary PC/Chol mixtures can be quite small, and is in agreement with the lack of $\mathrm{L}_{\mathrm{o}} / \mathrm{L}_{\mathrm{d}}$ heterogeneity observed in this type of mixture by confocal fluorescence microscopy [29].

\section{Visualizing membrane lateral heterogeneity: Fluorescence microscopy and atomic force microscopy}

In the last 10 years, techniques that allow collection of spatially resolved information, such as fluorescence microscopy (FM) and atomic force microscopy (AFM), have been applied to study membranes, opening new possibilities to explore further the lateral structure of lipid bilayers. These techniques provide spatial resolutions that range from nano-(AFM) to micrometers (FM). In the case of AFM the use of supported membranes (monolayers and/or bilayers) is required and the use of reporters (probes) is not needed. The latter technique provides nanometer lateral resolution and Ångström resolution vertically allowing visualization of phase separated lipid domains in planar membranes [30]. On the other hand, FM and related techniques (such as fluorescence lifetime imaging, fluorescence correlation spectroscopy, anisotropy imaging) provide a lateral resolution limit of $\sim 300 \mathrm{~nm}$ and obviously require the use of fluorescent probes. Applications of FM techniques [31] can be done on giant free standing-membranes (giant unilamellar vesicles - GUVs) as well as supported-membranes (monolayers and/or bilayers). Studies reporting physical features of lipid domains (such as shape, height, size, local order and time evolution) have been reported for several lipid mixtures using FM related techniques [13,18,29,31-33] and AFM [34-37]. Also the physical characterization of lipid domains was achieved in several cases by combining these two microscopy techniques [38-44].

The additional "visual" information obtained in GUVs/planar membrane experiments agrees and complements very well in many cases (although some discrepancies were reported, see below) with the main body of information obtained with the aforementioned classical approaches (NMR, DSC, ESR, fluorescence). For example, transition temperatures obtained in GUVs composed of binary mixtures agree very well with those reported in phase diagrams obtained from NMR experiments [31,45]. Van Duyl et al. [46] described domain formation in a DOPC/SM/Chol mixture by a combination of ${ }^{2} \mathrm{H}-\mathrm{NMR}$ and AFM.

Fluorescence microscopy on GUVs has been recently used to determine phase diagrams for canonical raft mixtures $[13,47]$. To our knowledge no information exists at present about construction of phase diagrams for bilayers composed of raft mixtures using AFM. Some discrepancies have been observed for the canonical raft mixture PSM/POPC/Chol phase diagram obtained from FM experiments (GUVs labelled with Texas Red-DPPE) [13] and from fluorescence spectroscopy experiments (e.g. FRET in large unilamellar vesicles - LUVsusing NBD-DPPE and Rhodamine-DOPE) [26] (see Section 9.2). Recently it was reported that lipid photooxidation under the microscope would affect the observed domains [48], and a study described that this is a general artifact observed at least in those cases when an unsaturated lipid was present (DOPC and POPC were studied) [21]. This would rule out all the microscopy work carried out up to now by the different groups, including studies by those authors. However, most of the other researchers have not reported such a severe effect, so in our opinion microscopy remains a very important methodology in this area. Other relevant points are related to the poor equilibration and compositional heterogeneity of the GUV, although variations in vesicle morphology following changes as low as 0.02 in cholesterol molar fraction are reported [21].

However, it is important to remark that most of the GUV experiments used to determine phase diagrams are solely based on the acquisition of fluorescence intensity images at different lipid compositions and temperatures [13,29]. No additional quantitative information beside the determination of either i) the temperature where (micrometer sized) domains emerge in the membrane, or ii) the lipid composition where (micrometer sized) lipid domains exist are generally exploited to build phase diagrams. Additionally, the fluorescence microscopy images are generally obtained using fluorescent molecules where the partition between ordered and disordered phases depends on the chemical composition of the lipid domains and not on the actual domain phase state [31]. Thus generalizations of the fluorescent molecule's affinity for the different lipid phases can be problematic. In these cases either an extensive characterization of the probe partition into domains [49] or measurements of other (fluorescent) parameters of the reporter (fluorescence lifetime, anisotropy, diffusion coefficient, emission spectral shift; see for example [18,31-33]) are highly recommended to obtain more reliable information about the actual "phase" of lipid domains in each particular lipid mixture. More surprising is the fact that dedicated image analysis has not been applied so far to determine the relative fractions of the coexisting phases present (neither in GUVs nor in planar membranes) for phase diagram determination. This very important information will certainly aid in validating the accuracy of the reported phase diagrams from GUVs. Additionally, by knowing the different lipid phase area fractions the lever rule can be tested. The latter allows confirmation that the membrane achieves thermodynamic equilibrium (something that has not been strictly demonstrated so far in GUV experiments). Some efforts in this direction are being presently performed in one of our laboratories [50]. With respect to the above mentioned discrepancies in the reported PSM/POPC/Chol phase diagrams, a practical solution is to apply the same experimental approach to both systems, e.g. FRET experiments in GUVs using the same fluorophores as in the FRET experiments done in LUVs. This is at present technically possible, but no such comparative study has been reported yet.

At this point it is obvious that the potentialities of the available microscopy techniques have not been fully exploited yet for membrane systems. Even though substantial new information was 
obtained in the last ten years using microscopy techniques, more rigorous strategies must be implemented (particularly for the construction of phase diagrams with information obtained from these techniques). It is also clear that the combination of AFM and FMrelated techniques applied to membrane systems will emerge as an attractive experimental approach. Stimulated emission depletion microscopy-STED-with a resolution of $30 \mathrm{~nm}$ will also be an obvious candidate here [51]. The latter has the unique advantage of bridging membrane structure (morphology/topology) with dynamics at molecular and supramolecular levels [33].

\subsection{Correlation between compositionally complex membranes and canonical raft mixtures}

It is important to realize that compositionally complex membranes present a much greater analytical challenge than the relatively simple membrane systems mentioned above. For instance, in a complex membrane that consists of more than three components, it is not possible to build up a phase diagram in order to obtain detailed information about the phase coexistence scenario, and this limitation makes comparison with the compositionally simple case rather difficult. One means of circumventing this problem is to use experimental techniques that may be combined with data obtained from measurable physical characteristics of the membrane in order to provide visual information about membrane lateral heterogeneity [31,45,52-55]. This information offers a way to link the compositionally simple and complex cases when direct use of the 'bulk' techniques mentioned above is not feasible. This approach was recently used in several studies in which data from either fluorescence microscopy [38,56-59] or atomic force microscopy $[38,42,58]$ provided a visual correlation of the lateral structure among model systems composed of simple lipid mixtures, natural lipid extracts and native membranes.

Although there are a variety of analytical approaches that may be used to characterize a membrane system, sometimes data derived from different techniques do not agree, e.g. in the determination of the size of membrane rafts. It is commonly accepted that raft size in biological membranes is of the order of tens to hundreds of nanometres, thus not resolvable by conventional microscopy techniques. ${ }^{1}$ However, there are published examples of biological systems that display membrane domains with sizes in the order of micrometers; these data are not generally quoted in the raft literature, but are consistent with the lateral phase separation that is observed in some lipid model mixtures [13,32,56,60]. For example, transient micron-sized regions were reported by Gaus et al. [61] in living macrophages using the fluorescence probe LAURDAN and two-photon excitation fluorescence microscopy. This study demonstrated the presence of microscopically visible lateral phase separation in living cells, strongly supporting the notion that cholesterol had an effect on lateral organization in the membrane under study. Interestingly, this result is consistent with a report by Gousset et al. [62] in which micron-sized domains were also observed in activated platelets. In the latter study, lipid mixtures with defined compositions ( $\mathrm{POPC} / \mathrm{SM} / \mathrm{Chol}$ ) based on the abundance of the major lipid components of the platelet membrane were used to mimic the lateral heterogeneity observed in this biological membrane [62,63]. An additional interesting investigation in this area was carried out by Bernardino de la Serna et al. [38]. These authors examined the lateral behaviour of native pulmonary surfactant membranes (bilayers), which contain high amounts of

\footnotetext{
${ }^{1}$ It is important to mention that LAURDAN GP experiments performed in GUVs using linear polarized light as an excitation source can be exploited to obtain information about coexistence of lipid domains with sizes below the resolution of the microscope (the resolution of the microscope is $\sim 250 \mathrm{~nm}$ radial), see Bagatolli and Gratton [53] and references therein.
}

the saturated phospholipid DPPC, together with cholesterol, unsaturated phospholipid and a low percentage of membrane proteins. Based on the information obtained in POPC/DPPC/Chol model systems [64], the ratio of cholesterol with respect to phospholipids (DPPC and unsaturated lipids) found in pulmonary surfactant membranes suggests the possible presence of phase coexistence under physiological conditions. This was confirmed by Bernardino de la Serna et al. [38], who demonstrated that the native material at physiological temperatures displayed a fluid-ordered/fluid-disordered-like phase coexistence (Fig. 3). In these experiments, GUVs (giant unilamellar vesicles) composed of native pulmonary surfactant membranes were compared with GUVs composed of the ternary mixture DOPC/DPPC/Chol (see Veatch et al. [64], for a phase diagram for such a lipid mixture). Interestingly, in the pulmonary surfactant system, extraction of the surfactant membrane proteins did not change the observed lateral organization of the native membrane. However, cholesterol extraction from the native material using methyl- $\beta$-cyclodextrin changed the lateral structure from fluid-ordered/fluid-disordered-like phase coexistence into a gel/fluid-like phase coexistence, as was also observed in an analysis of BLES, a clinical pulmonary surfactant that lacks cholesterol [57]. Taken together, these data indicate that lipids play a crucial role in regulating the lateral structure of the pulmonary surfactant membranes. The relationship between membrane lateral heterogeneity and membrane function that was reported in this work suggests that lateral phase separation is likely to be important for the proper functioning of these membranes [38]. The experimental approach described above can also be extended to other membranous systems, as was demonstrated by Ruan et al. [65]. In particular, the recent availability of GUVs containing the full composition of native biological membranes [66,67] is opening exciting possibilities to proceed with this type of study.

\section{Lipid monolayers: mechanical and optical measurements}

Lipid monolayers, studied in situ or after transfer to solid supports (i.e. supported monolayers), are an indispensable tool for understanding the behaviour of lipids in membranes. Many physical phenomena displayed by lipids in biomembranes can also be observed and modelled in lipid monolayers. These phenomena, which reflect the chemical and structural features of different lipids, include phase transitions, changes in lateral diffusion, alterations in lateral compressibility/elasticity, and mixing interactions that can result in critical points as well as immiscible or coexisting lateral phases (domains) [12,68-71]. Thus, monolayer studies have not only provided insights into phase behaviour of raft lipids but also have yielded fundamentally important data regarding the essential roles played by acyl chain structure (saturation vs. unsaturation) and the sphingoid base in promoting lateral interactions that occur between various 'raft' lipids [72-76]. One noteworthy conclusion was that sphingomyelin possessed subtle but important features not present in the "model raft lipid', DPPC, even though both lipids contain saturated acyl chains. Also, cholesterol addition (0.3-0.5 mol fractions) was found to lower the lateral elasticity of the naturally-prevalent PC motif of saturated $s n-1$ and unsaturated sn-2 acyl chains (e.g. POPC) much less than in DOPC, another popular 'model raft lipid'.

Although early monolayer studies involving construction of lipid phase diagrams were limited to mixtures consisting of only two different lipid components $[69,70,77]$, more recently direct insights into the monolayer phase behaviour of raft ternary mixtures of PC/SM/ Chol have been stimulated by using epifluorescence microscopy to monitor the distribution patterns of trace amounts of lipids containing covalently attached reporter fluorophores [57,78-80]. Also, because this same combination of lipids can form stable giant unilamellar vesicles (GUVs, see Section 3), direct comparison of lipid mixing, domain formation, and construction of ternary phase diagrams has 

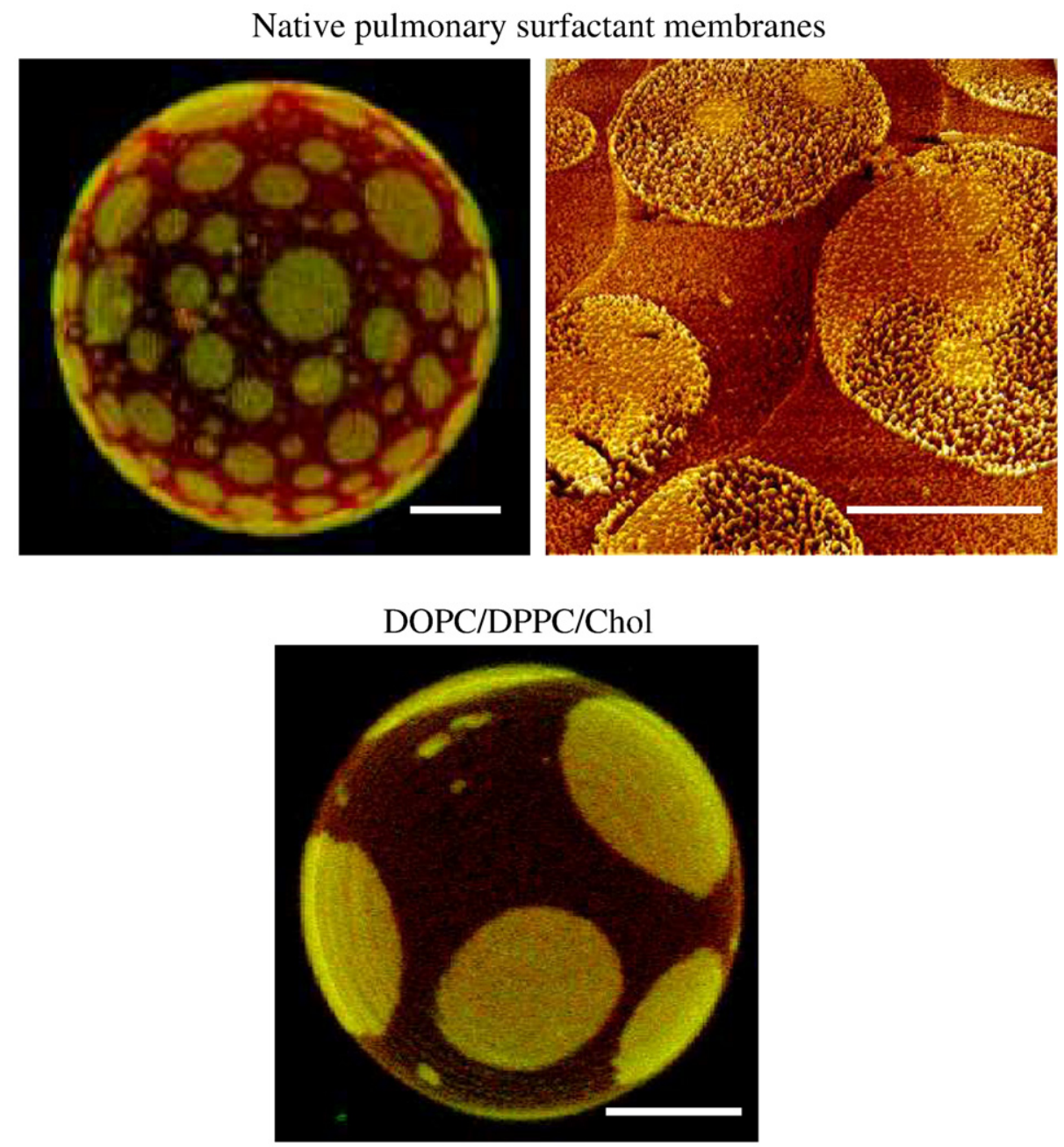

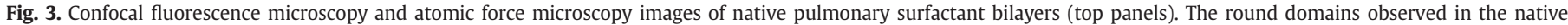

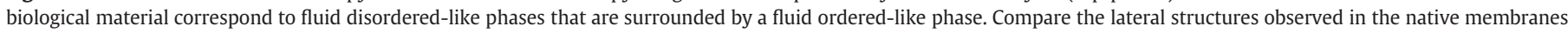

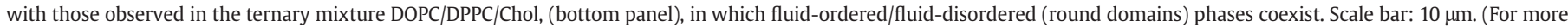
information see [38]).

been possible in bilayers (GUVs) and in monolayers using epifluorescence microscopy. Not surprisingly, interesting similarities and differences have emerged.

In monolayers, ternary mixtures of $\mathrm{POPC} / \mathrm{PSM} / \mathrm{Chol}$ produce two distinct regions of immiscible liquid phases: a cholesterol-diminished $\alpha$-region and a cholesterol-enriched $\beta$-region. Within the $\alpha$ region, dark domains are observed on a bright background and the amount of the dark phase increases with increasing cholesterol concentrations, suggesting that the fluorescent dye used as a tracker (0.5 mol\% Texas Red-DPPE) partitions less readily to the cholesterolrich phase that constitutes the $\beta$-region. Within the $\beta$-region, sparsely distributed, bright domains are evident, consistent with the notion that the dye preferentially distributes into the cholesterol-poor $\alpha$-region [79]. The $\alpha$-region is easily identified by using fluorescence microscopy, because as the surface pressure is lowered, the uniform monolayer abruptly separates into two coexisting liquid phases. The transition from $\alpha$-to $\beta$-region is more difficult to identify, because at high pressures, the contrast often changes from bright domains on a dark background to the opposite, presumably because of the pressure-dependent, lateral transfer of the fluorescent lipid probe from the lipid domains into the surrounding fluid lipid phase. As the surface pressure is lowered below $5-15 \mathrm{mN} / \mathrm{m}$, uniformly dispersed small bright domains appear and join the preexisting sporadically scattered bright domains.
Such visual criteria can be used to construct ternary phase diagrams that allow a comparison of the miscibility and phase behaviour in monolayers and bilayers at $25{ }^{\circ} \mathrm{C}$ (Fig. 4). In GUVs, micron-size coexisting liquid domains are limited to the shaded region shown in Fig. 4. In contrast, micron-size liquid domains are evident in monolayers of all of the tested compositions, including the binary axis of cholesterol mixed with either POPC or PSM. It is noteworthy that the boundary for domain coexistence in GUVs at $25{ }^{\circ} \mathrm{C}$ spans both the $\alpha$ - and $\beta$-regions of the monolayer. Within the monolayer $\alpha$-region, micron-size liquid domains are observed only below $\sim 14 \mathrm{mN} / \mathrm{m}$ (milli-Newton per meter; a measure of surface tension) although it should be noted that this observation does depend on the lipid mixing ratios. This surface pressure range is well below the $30-35 \mathrm{mN} / \mathrm{m}$ level that is thought to produce lipid packing conditions similar to those found in biomembranes (see, for example, [80bis,81,82]). Interestingly, in monolayers formed from total lipid extracts of human erythrocytes, epifluorescence microscopy revealed the presence of two immiscible liquid-like domains at surface pressures above $20 \mathrm{mN} / \mathrm{m}$ [83]. Lipid compositions simulating the outer leaflet of the red cell membrane have a miscibility critical point near $29 \mathrm{mN} / \mathrm{m}$, which is a surface pressure that produces monolayer lipid densities comparable to those in the erythrocyte membrane.

Also yielding valuable insights into monolayer lipid mixing behaviour is a variation of the in situ approach involving transfer of 


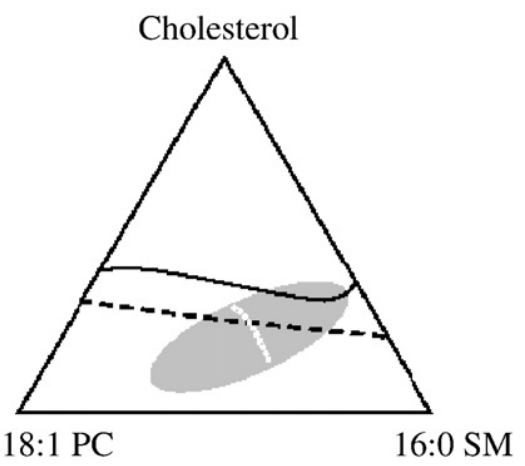

Fig. 4. Miscibility in monolayers vs. GUVs. The dashed black and the white lines denote the change in contrast from dark backgrounds with bright domains to light backgrounds with dark domains in monolayers and GUVs, respectively. The solid black line denotes the transition from the $\alpha$-region to the $\beta$-region in monolayers. The gray shaded region indicates the region of coexisting liquid phases in GUVs. This figure is adapted from [79].

the monolayers from the air/water interface to alkane-coated, solid supports (i.e. supported monolayers) for study by various techniques. While the basic strategy is well-established for mapping phase diagrams in mixed lipid monolayers (e.g., [68]), applications to ternary raft lipid combinations have emerged only recently in combination with epifluorescence microscopy [56,60], atomic force microscopy [84-86] and/or imaging mass spectrometry. The latter approach, used by Winograd and colleagues [14,87-89], provides an exciting new means for direct imaging of the mixing behaviour of complex lipid combinations in the absence of fluorophore probes that can alter microdomain morphology. Moreover, the imaging mass spectrometry approach enables lipid identification and quantitative estimates of different lipid components within differing domains. Ternary mixtures of PC/SM/ Chol, have been analyzed in detail, confirming formation of domains enriched in sphingomyelin and cholesterol as well as the importance of acyl composition in controlling domain formation [14,89].

Another promising development for elucidation of the micro- and nanomixing behaviour of complex mixtures of lipids, including raft lipid mixtures, involves in situ measurement of fluorescence emission spectra in monolayers [90]. Although not yet reported for raft lipid mixtures, this approach has the potential for detection at nanodomain resolution limits. The latter potentiality can be expanded by implementing other fluorescence microscopy related techniques (described in Section 3) in monolayers at the air-water interface. The latter will provide simultaneous structural and dynamic information not presently available for this system.

\section{Detergent solubilization studies}

SM/Chol mixtures are extremely resistant to solubilization by nonionic detergents [91], and solubilization may result in the formation of a detergent-resistant membrane fraction. Such non-solubilized remnant fractions have been identified, sometimes incorrectly, as membrane rafts [19]. Therefore a discussion of the detergent solubility of SM, PC, Chol, and their mixtures is relevant in this context. Bilayers composed of certain binary or ternary mixtures of SM and Chol, with or without PC, exhibit substantial composition-dependent differences in their susceptibility to solubilization by detergents. The non-ionic surfactant Triton X-100 is by far the most commonly used detergent in these studies, and the observation that some of these mixtures, particularly those rich in SM and Chol, resist solubilization even at high Triton X-100 concentrations may be relevant in the search for a physico-chemical explanation of the phenomenon of 'detergentresistant membranes' [92-95].

Bilayers composed of pure PC in the fluid (liquid-disordered) state are fully solubilized at effective detergent/lipid molar ratios of $\sim 3.5: 1$
[96,97]. For a precise definition of the term 'effective detergent/lipid ratios' see Lichtenberg [97bis]; for methodological aspects in the study of membrane detergent solubilization, see Goñi and Alonso [98]. An analysis of $\mathrm{PC}$ in the gel state found that solubilization was highly dependent on the chain length. At $\sim 20^{\circ} \mathrm{C}$ below their respective gelfluid transition temperatures, dimyristoyl (C14) and dipentadecanoyl (C15) PC could be easily solubilized by Triton X-100, while the C16 and higher analogues were totally insoluble at a 5:1 detergent:lipid ratio [99]. For pure PC's in the fluid state, Ahyayauch et al. [100] found that acyl chain unsaturation increased the amount of Triton X-100 required to achieve solubilization, i.e. unsaturation made the PC bilayers more resistant to solubilization. Pure SM bilayers in the fluid state were more easily solubilized than pure PC bilayers under the same conditions, with full SM bilayer solubilization generally being achieved at a detergent/lipid ratio of 1.5:1 [101]. Chicken-egg SM, which has a gelfluid transition temperature of $\sim 39{ }^{\circ} \mathrm{C}$, was nearly insoluble at temperatures of $20{ }^{\circ} \mathrm{C}$ and below [91]. A detailed isothermal calorimetry study of Triton X-100 binding to pure SM bilayers at subsolubilizing detergent concentrations and temperatures ranging between 5 and $55{ }^{\circ} \mathrm{C}[102]$ revealed that $\Delta G$ of binding was virtually independent of the lipid phase. Thus the observed temperaturedependent changes in solubilization are due to phenomena occurring at stages beyond detergent binding.

The solubility of bilayers consisting of SM/PC binary mixtures increases as the proportion of SM in the mixture increases [101,103]. In general, the presence of the more complex sphingolipids such as gangliosides, facilitates the Triton X-100 solubilization of lipid bilayers (Sot et al., 2002). The binary mixture SM:Chol gives rise to detergentresistant bilayers under most conditions, and Patra et al. [101] found that $15 \mathrm{~mol} \%$ Chol rendered the SM/Chol bilayers totally resistant to solubilization by Triton X-100 at a 5:1 detergent:lipid molar ratio at $50{ }^{\circ} \mathrm{C}$. At $22{ }^{\circ} \mathrm{C}, \mathrm{SM} / \mathrm{Chol}$ bilayers became fully resistant to solubilization at Chol concentrations above $20 \mathrm{~mol} \%$; such cholesterol concentrations were reported to induce formation of a $\mathrm{L}_{0}$ phase in similar DPPC/Chol bilayers [10,104]. This is notable particularly because the $L_{o}$ phase has been shown to be intrinsically more resistant to detergent solubilization than the $L_{d}$ phase $[105,106]$. Patra et al. [101] also assayed the solubilization of binary mixtures of SM with various sterols, and results suggested that both the hydroxyl group at C3 and the hydrocarbon chain at C17 of the steroid nucleus appeared to be required to promote insolubility. Xu et al. [107] observed the concomitant formation of ordered domains and decreased solubilization of SM bilayers when certain sterols, including cholesterol, were incorporated into the mixture. Li et al. [108] similarly performed solubilization assays on SM:sterol mixtures and arrived at the same conclusions. The same authors $[76,108]$ have also shown that equimolar SM/Chol films have unusually low lateral elasticity, which could be an important physical feature of the $\mathrm{L}_{0}$ phase and may correlate with detergent resistance.

The Triton X-100 solubilization of PC/Chol binary mixtures has been examined in a number of studies. Early work by Urbaneja et al. [109] showed that when Chol was mixed at equimolar proportions with egg PC, there was a decrease in bilayer solubility at room temperature, although no preferential solubilization of one component over the other was observed. From the perspective of 20 years later, we suggest that in this early study, what was observed was the Chol-induced formation of the $\mathrm{L}_{\mathrm{o}}$ phase, thus leading to a decrease in solubility. Other work $[107,110,111]$ has measured the solubilization of DPPC/sterol bilayers by Triton X-100 at $23^{\circ} \mathrm{C}$. Results showed that some sterols such as cholesterol gave rise to detergent resistance, while others such as androstanol (which lacks the hydrocarbon chain) actually favoured DPPC solubilization. Simultaneous measurements of $\mathrm{L}_{o}$ domain formation enabled the authors of these studies to establish a correlation between tightly packed, ordered domain formation and resistance to Triton X-100 solubilization. Li et al. [76] reported an apparent correlation between lateral elasticity (the ease of 
or resistance to lateral compression as assessed by the surface compression modulus; see [112]) and resistance to solubilization by Triton X-100 in Chol/disaturated PC mixtures. In further work, Li et al. [108] noted the relevance of certain structural features of the Chol molecule, notably the isooctyl chain at C17, to the modulation of lateral compressibility and to the detergent-solubility of $\mathrm{PC} /$ Chol bilayers.

The solubilization of egg PC/egg SM/Chol ternary mixtures by Triton X-100 was examined by Sot et al. [91]. In these experiments, the egg PC proportion in the bilayer varied, while the SM/Chol equimolar ratio was kept constant, so that the mixtures had the general composition PC/SM/Chol in an N:1:1 molar ratio, with $N$ varying from 2.7 to 9.0. Results revealed the existence of three distinct regions of PC:SM:Chol composition with respect to detergent solubilization: (1) region I of mixtures that could not be solubilized even at a detergent: lipid molar ratio of 5:1, or at PC concentrations of less than $60 \mathrm{~mol} \%$ (which corresponded to $N \leq 3$ ), (2) region II of mixtures that could be solubilized, but required higher detergent concentrations than pure PC, or required PC concentrations in the $60-73 \mathrm{~mol} \%$ range (which corresponded to $3 \leq N \leq 5$ ) for solubilization, and (3) region III of mixtures that were solubilized under similar conditions as pure PC, with PC ranging from $73 \mathrm{~mol} \%$ and above (which corresponded to $N \geq 5.5$ ). These data suggest that regardless of what the interaction between SM and Chol that gives rise to detergent resistance is, such interaction is lost upon the dilution of SM and Chol with PC. The effect of fatty acid unsaturation on Triton X-100 solubilization of ternary mixtures was explored by Stillwell and co-workers [113,114], in SM/PE/ Chol mixtures, in which the fatty acid composition of PE was changed from palmitic (C16:0) to docosahexaenoic (C22:6). Increasing unsaturation resulted in increased solubilization, suggesting that the unsaturated PE-enriched fraction tends to separate from the SM/ Chol-rich domains more than the saturated one.

The solubilization by Triton X-100 of a SM/PC/Chol ternary mixture was monitored by Heerklotz [115] using differential scanning calorimetry, pressure perturbation calorimetry, and isothermal titration calorimetry. The results suggested that addition of Triton X-100 could create ordered domains in an homogeneous fluid membrane which would be, in turn, detergent-resistant upon subsequent membrane solubilization. Further comparison with pure POPC membranes reveals a very unfavourable interaction between Triton X-100 and SM/Chol, which causes a substantial tendency to separate SM-rich detergentresistant-like and Triton-rich fluid domains [116]. Based on these and related experiments, Keller et al. [117] have proposed a quantitative model describing the selective solubilization of membrane domains.

The issue of $\mathrm{L}_{o}$ phase resistance to detergent solubilization, first demonstrated for the DMPC/Chol mixture [105], has been recently examined for the $\mathrm{PC} / \mathrm{SM} / \mathrm{Chol}$ composition using atomic force and confocal fluorescence microscopy [34]. The authors observe a preferential solubilization of lipids in the $L_{d}$ over those in the $L_{o}$ phase for Triton X-100 and CHAPS, but not for octylglucoside, with Lubrol and Brij 96 showing an intermediate behaviour. This confirms, once again, the observation that different detergents follow different solubilization patterns (if not necessarily different solubilization mechanisms).

\section{Probe-free methods: calorimetric and X-ray studies}

\subsection{Calorimetric studies}

Most naturally occurring SMs exhibit thermotropic transitions from the 'lamellar gel' to the 'lamellar liquid crystalline' states at $T_{\mathrm{m}} \approx 30-40{ }^{\circ} \mathrm{C}$ [118-120]. This is commonly called the gel-fluid transition, and it can be conveniently monitored by DSC as well as by other methods. Although the disaturated PCs also undergo a similar transition, the mono- and polyunsaturated species that are commonly found in cell systems do not. The SM gel-fluid phase transition is very sensitive to the presence of other lipids in the bilayer, and its associated thermodynamic parameters provide information on the properties of the lipid mixture, such as ideality or heterogeneity. Therefore, DSC is very useful in the context of the phase studies of PC/ $\mathrm{SM} /$ Chol systems.

Mixtures of egg PC and bovine brain SM have been studied by Untracht and Shipley [118], and results showed that at $44{ }^{\circ} \mathrm{C}$ (i.e. above the SM transition temperature), both lipids were miscible at all tested proportions, while at $20{ }^{\circ} \mathrm{C}$ (i.e. below the $T_{\mathrm{m}}$,), a liquid crystalline phase that consisted mainly of PC separated from one or more of the gel-like phases enriched in SM; this effect was particularly prominent for SM concentrations above $33 \mathrm{~mol} \%$. More recent studies by RuizArgüello et al. [121] using egg PC and egg SM confirmed the above observations. A temperature-composition diagram (phase diagram) included in the latter study showed that egg PC was partially miscible with egg SM in the gel state, and that this miscibility led to a progressive decrease in the onset of the phase transition. Similar results were later described by Pokorny et al. [122] in a BSM/POPC system. Calhoun and Shipley [119] used DSC to study the interaction between PSM and DMPC, both of which are fully saturated lipids, and found good miscibility in both the gel and fluid phases, with no lateral phase separation. This was attributed to the relatively close $T_{\mathrm{m}}$ of both lipids $\left(40{ }^{\circ} \mathrm{C}\right.$ for SM and $23^{\circ} \mathrm{C}$ for PC); no lateral phase separation was found either in mixtures of DPPC $\left(T_{\mathrm{m}}=41^{\circ} \mathrm{C}\right)$ with DMPC. However, years later this work was expanded by Térová et al. [123], who studied the miscibility of different saturated PC with PSM. Based on criteria of the ideality of mixing and the degree of cooperativity of the main transition, these authors concluded that DMPC, dipentadecanoyl PC, and 1-myristoyl-2-palmitoyl PC interacted most favourably with PSM in bilayer membranes, while DPPC showed much less ideal miscibility, in spite of the identical fatty acyl chain length and very similar $T_{\mathrm{m}}$.

The effects of Chol on egg SM in bilayer membranes have been studied in detail by Mannock et al. [124]. In this and in other similar studies [119,125-127], results showed that cholesterol caused the SM transition endotherm to broaden, and caused its enthalpy to decrease without inducing any marked changes in the $T_{\mathrm{m}}$ midpoint transition temperature. At Chol concentrations of about $50 \mathrm{~mol} \%$, the transition is abolished, and the system exists in a $\mathrm{L}_{\mathrm{o}}$ phase at all temperatures studied. In mixtures containing up to $20 \mathrm{~mol} \% \mathrm{Chol}$, the corresponding thermograms can be deconvoluted into a sharp component and a broad component, which are interpreted as arising from the melting of cholesterol-poor and cholesterol-rich domains, respectively. Although studies on SM/Chol systems containing very high Chol proportions (e.g. 60-80 mol\%) are rare, Epand [128] found that in membrane systems containing these high Chol ratios, anhydrous or monohydrate Chol crystallites formed with a $T_{\mathrm{m}}$ of $80-95{ }^{\circ} \mathrm{C}$.

A recent publication [129] describes the properties of DPPC/Chol mixtures using a combination of molecular acoustics techniques with DSC and pressure perturbation calorimetry. As revealed by detailed measurements of the temperature and concentration dependence of the isothermal compressibility and thermal expansion coefficient of the DPPC/Chol system, the data show no evidence for the existence of a critical point phenomenon at Chol concentrations ca. $22 \mathrm{~mol} \%$, above which only a $\mathrm{L}_{\mathrm{o}}$ phase is supposed to exist [130]. Krivanek et al. [129] propose, in agreement with earlier suggestions [131-133] that the DPPC/Chol system in the $\mathrm{L}_{0}+\mathrm{L}_{\mathrm{d}}$ coexistence region observed in the phase diagrams $[130,134]$ should be described, on a macroscopic level, as a largely homogeneous phase, consisting of small nanodomains. At present, even the concept of a $L_{o}$ phase is a matter of debate. The observations by Krivanek et al. will undoubtedly contribute to the discussion.

DSC studies of three-component SM/PC/Chol mixtures are considered next. In an early study, Calhoun and Shipley [119] examined mixtures of Chol with PSM and DMPC (1:1 molar ratio), and found that the effects of cholesterol on the phospholipid mixture were very similar to those observed on the individual lipids. The corresponding endotherms could be deconvoluted into a sharp component and a broad component: The enthalpy associated with the sharply melting 
component decreased with increasing cholesterol, reaching zero at a Chol mole fraction of $0.20-0.25$, while the $T_{\mathrm{m}}$ of this component remained invariant at $32{ }^{\circ} \mathrm{C}$. The broad component remained visible even at a $0.33 \mathrm{~mol}$ fraction of $\mathrm{Chol}$, although it did extend over $50{ }^{\circ} \mathrm{C}$, and the $T_{\mathrm{m}}$ of the broad component appeared to be shifted toward higher temperatures with increasing Chol content. More recently, in the related system PSM/PE/Chol, Wassall and co-workers [114,135] could confirm that Chol abolished the gel-fluid transition of the phospholipids. In addition, using PE with a varying degree of unsaturation, they concluded that phospholipids with polyunsaturated fatty acids were excluded from SM/Chol-rich domains. Dietrich et al. [56] used GUVs and fluorescence microscopy and observed a presumed $\mathrm{L}_{\mathrm{o}}$ to $\mathrm{L}_{\mathrm{d}}$ transition at $25{ }^{\circ} \mathrm{C}-30{ }^{\circ} \mathrm{C}$ in equimolar DOPC/BSM/Chol mixtures. Gandhavadi et al. [136] studied the same mixture by DSC, but could not detect any transition in the $3{ }^{\circ} \mathrm{C}-70{ }^{\circ} \mathrm{C}$ range. It is, however, important to note that certain thermotropic transitions that occur with a low cooperativity cannot be detected by DSC. No transition was detected either by Pokorny et al. [122] in BSM/POPC/Chol for mixtures containing equimolar amounts of BSM/Chol, irrespective of POPC concentration. For mixtures with widely differing proportions of BSM and Chol, eg. 2:1, or 3:1, complex thermograms were observed, often extending to higher temperatures than BSM alone. This may suggest that $1: 1$ is a critical SM/Chol ratio in mixtures with POPC.

A different approach was taken by Tsamaloukas et al. [137], who performed a comparative analysis of the interaction of Chol with POPC or $\mathrm{SM}$ in fluid phase, ternary bilayers at $50{ }^{\circ} \mathrm{C}$, using isothermal titration calorimetry. Chol was solubilized with methyl- $\beta$-cyclodextrin, and the uptake of Chol into, or release from, the vesicle bilayers was measured. Chol affinity for SM was estimated to be 5-fold higher than for POPC. Bringing Chol in contact with SM was highly exothermic, compared to POPC. That SM/Chol interactions are enthalpically favourable implies that the preference of Chol for SM increases with cooling, and can induce domain formation below a certain temperature. Thus temperature may be an important factor in the observation of nano-or macrodomains consisting of SM/Chol in the $\mathrm{L}_{\mathrm{o}}$ phase.

\subsection{X-ray studies}

The structure of lipid phases has been defined from X-ray diffraction studies (see [138] for a review of the early studies). The dimensions of the various phases, $\mathrm{L}_{\beta}, \mathrm{P}_{\beta^{\prime}}, \mathrm{L}_{\alpha}, \mathrm{H}_{\text {II }}$, etc. were described by Luzzati and co-workers in the sixties of the past century. X-ray diffraction was applied by Calhoun and Shipley [119], in the study mentioned above in connection with DSC, to pure PSM, PSM/DMPC, PSM/Chol, DMPC/Chol, and PSM/DMPC/Chol. These authors described the lamellar phases formed by these lipids long before the "raft" concept was proposed. Later Shipley and co-workers [139] described the change in bilayer thickness of SM lamellae with increasing $\mathrm{N}$-acyl chain length (from C16:0 to C24:0), and suggested chain interdigitation when the length of the $\mathrm{N}$-acyl chain exceeded significantly $(\geq \mathrm{C} 20)$ that of the sphingosine chain.

As a result of the proposal of the "raft" hypothesis, a series of studies were published in which X-ray techniques were applied to the relevant lipid mixtures. Gandhavadi et al. [136] studied equimolar PC/ $\mathrm{SM} /$ Chol mixtures, and found a single liquid-crystalline phase. This was in contrast to, e.g. Dietrich et al. [56], who had reported lateral separation of liquid phases in a very similar mixture, observed by fluorescence microscopy. (Veatch and Keller [140] suggested that the equimolar mixture at room temperature was close to the miscibility transition temperature and that small changes in vesicle composition would have a large effect on the observed phase behaviour). Further data characterized in detail the $\mathrm{L}_{\mathrm{o}}$ phase as seen by X-ray diffraction in SM/Chol [141] and saturated PC/Chol [142].

Three coexisting phases, $\mathrm{L}_{\beta}, \mathrm{L}_{0}$ and $\mathrm{L}_{\mathrm{d}}$ were detected by synchrotron X-ray diffraction in DPPC/DOPC/Chol MLV's by Quinn and co-workers [143]. These authors estimated that Chol was almost equally distributed in the $L_{\beta}$ and $L_{d}$ phases. Chol molecules represented only $10 \%$ of the total molecules in these two phases, but they comprised $30 \%$ of the molecules in the $\mathrm{L}_{\mathrm{o}}$ phase.

Very recently, Nagle and co-workers [144] used wide-angle X-ray scattering from oriented lipid multilayers to distinguish the chain ordering in $\mathrm{L}_{\mathrm{o}}$ phases from that in $\mathrm{L}_{\mathrm{d}}$ phases. With aligned multilayers, the wide-angle, chain-chain diffraction from a conventional fluid $\left(L_{d}\right)$ phase is centred on the equator but spreads out in a broad arc towards the meridian, [145]. For an $\mathrm{L}_{\mathrm{o}}$ phase, on the other hand, the chain scattering intensity is concentrated much more on the equator, corresponding to the pronounced chain ordering by Chol [144]. Fig. 5 shows the dependence of the radially integrated chain diffraction intensity on the angle of arc, $\phi$, away from the equator, for DPPC multilayers with and without Chol at $45{ }^{\circ} \mathrm{C}$. In the $\mathrm{L}_{\mathrm{d}}$ phase without $\mathrm{Chol}$, the normalised intensity extends over a wide range of $\phi$, whereas in the $\mathrm{L}_{\mathrm{o}}$ phase with $40 \mathrm{~mol} \% \mathrm{Chol}$, the intensity is restricted to low values of $\phi$. The solid lines in Fig. 5 are fits of the intensity distribution to a model in which bundles of straight, hexagonally packed chains are oriented according to the Maier-Saupe pseudopotential $\left(V=m \cos ^{2} \beta\right.$, where $\beta$ is the angle to the director) that is used frequently to describe the ordering of nematic liquid crystals. The strength, $m$, of the orientational potential allows calculation of the corresponding order parameters, $\left\langle\mathrm{P}_{2}(\cos \beta)\right\rangle=\left(3\left\langle\cos ^{2} \beta\right\rangle-1\right) / 2$, which are given in the inset to

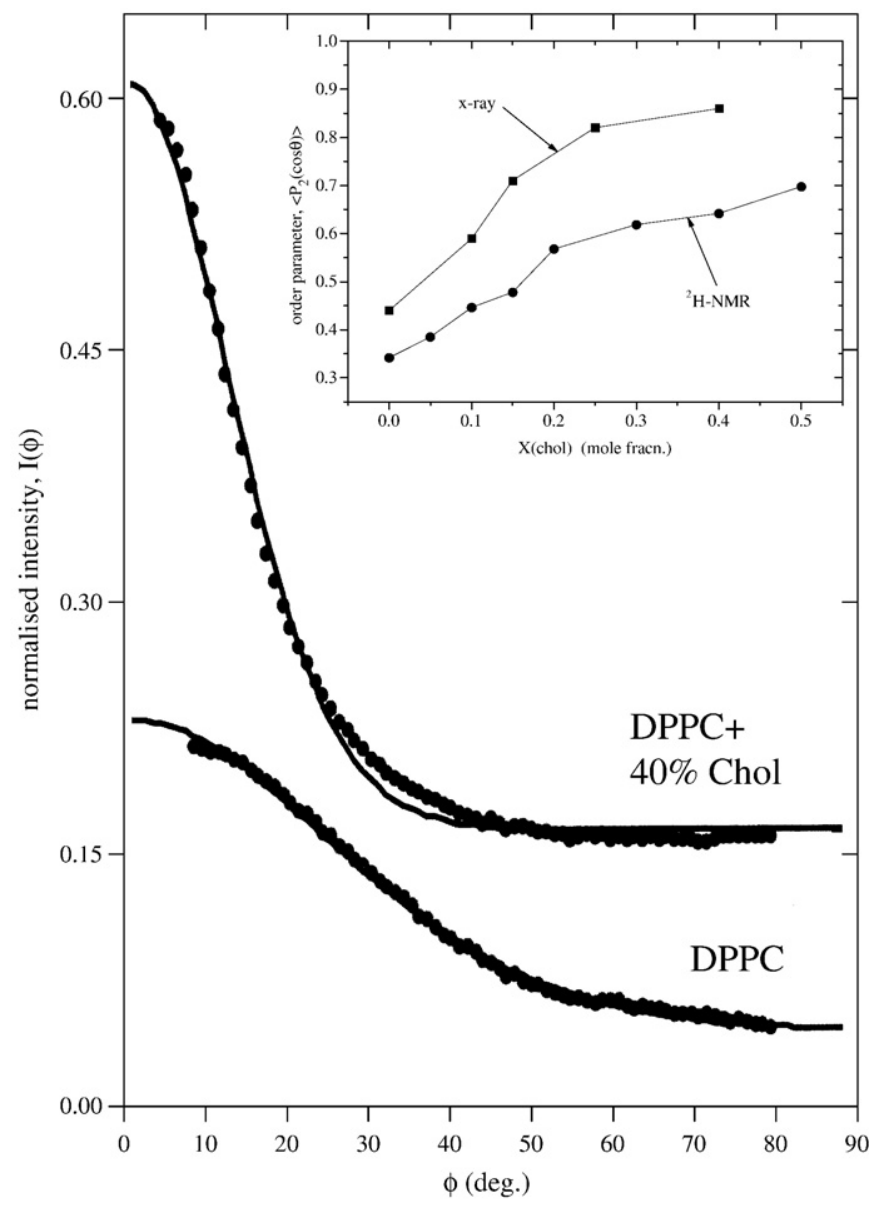

Fig. 5. Angular dependence of the wide-angle X-ray scattering intensity, $I(\phi)$, from aligned DPPC multilayers, with and without $40 \mathrm{~mol} \%$ cholesterol, at $45^{\circ} \mathrm{C}$ [144]. (Note: $I(\phi)$ for DPPC + Chol is offset from zero by +0.15 .) Black lines are fits to a single distribution of chain orientations, based on the Maier-Saupe potential of strength $m: I(\phi) \propto \exp \left(\frac{1}{2} m \cos ^{2} \phi\right) \times I_{0}\left(\frac{1}{2} m \cos ^{2} \phi\right)$, where $I_{0}$ is a modified Bessel function of the first kind. Inset: dependence on cholesterol content of the chain order parameters determined from the angular fitting of the X-ray intensities ( $m=11.3 \pm 0.4$ and $3.0 \pm 0.01$ for DPPC with and without Chol, respectively), or from ${ }^{2} \mathrm{H}-\mathrm{NMR}$ of the perdeuterated lipid chains [147]. 
Fig. 5. Not surprisingly, in view of the model used, these X-ray values differ from the average chain order parameters from ${ }^{2} \mathrm{H}-\mathrm{NMR}$, which are obtained from the individual $C_{2}$ segmental order parameters $S_{C D}$ $\left(S_{\text {mol }}=2\left\langle\left|S_{\mathrm{CD}}\right|\right\rangle\right)$, but they display the same dependence on membrane cholesterol content as do the NMR order parameters.

For binary mixtures of either DPPC or DOPC with cholesterol, no evidence of phase heterogeneity was found in the wide-angle scattering from aligned membranes in the fluid regime [144,146]. However, for $1: 1 \mathrm{~mol} / \mathrm{mol}$ mixtures of DOPC and DPPC with Chol contents of 15 to $30 \mathrm{~mol} \%$, the angular dependence of the chain diffraction intensity could not be fit by assuming a single distribution of chain orientations as in Fig. 5. Instead, it was necessary to assume two independent distributions, with associated order parameters that correspond to coexisting $\mathrm{L}_{\mathrm{o}}$ and $\mathrm{L}_{\mathrm{d}}$ phases, in order to get a good fit to the $\phi$-dependence of the wide-angle scattering intensity. This new X-ray criterion for fluid-fluid phase coexistence was used to determine the miscibility transition, which was found to occur at $T_{\text {mix }} \approx 35$ and $25{ }^{\circ} \mathrm{C}$ for cholesterol contents of 15 and $30 \mathrm{~mol} \%$, respectively, for the DOPC:DPPC 1:1 mol/mol mixture. In most cases, phase coexistence was also evidenced by the appearance of two sets of Bragg reflections in the small-angle region, corresponding to different bilayer repeat spacings. It is the order parameters from the accompanying wide angle scattering, however, that identifies these as coexisting $\mathrm{L}_{\mathrm{d}}$ and $\mathrm{L}_{\mathrm{o}}$ phases. Although the systems studied so far by the wide-angle X-ray method do not contain sphingomyelin, DPPC can be considered as a surrogate for sphingomyelin in this context.

A further scattering method, namely small-angle neutron scattering (SANS) has been applied by Katsaras and co-workers to the detection of submicron-sized raft-like domains in small unilamellar vesicles $[148,149]$. Neutron scattering is very sensitive to differences between hydrogen and deuterium, thus domains that are enriched in a given phospholipid can be selectively labelled using a chaindeuterated form of that phospholipid.

\section{NMR spectroscopy}

NMR has been used extensively since the 1970s to probe the organization of lipids in membranes. Since no sterically intrusive probes are required, this technique has found favour in studies of membrane thermotropic behaviour. Probes, even at low concentrations, may provide misleading information on phase transitions if their preferential partitioning between the different phases is not taken into account (see Section 2.1). Recently it was observed that the temperature at which ternary lipid mixtures displayed micron-sized liquid/liquid domain coexistence was exquisitely sensitive to the probe concentration [150]. Deuterium $\left({ }^{2} \mathrm{H}\right)$ NMR using chain-labelled lipids can provide a quantitative measurement of coexisting phases within a membrane (see [151] for a detailed description of this technique), and in liquid crystalline bilayers, ${ }^{2} \mathrm{H}$ NMR spectra accurately reflect the conformational disorder of hydrophobic chains (Fig. 6). ${ }^{31}$ P NMR monitors the headgroup region of phospholipids and yields spectra that have shapes specific to particular membrane phases. Pulsed field gradient (PFG) NMR experiments have been used to measure lipid-specific diffusion in mixed-lipid membranes and can distinguish whether lateral motions are occurring in a homogeneous membrane or in one with coexisting liquid crystalline domains. For a recent review of this technique, see [152]. The membrane solubility of a given lipid can also be measured using NMR, and this is particularly useful for determining the extent to which Chol can be incorporated into membranes before crystallizing out as Chol monohydrate within the 'inaccessible top' of the raft mixture triangular phase diagram.

\subsection{Pioneering work on binary lipid systems: the liquid-ordered phase}

The $L_{o}$ phase was first proposed by Ipsen et al. [10], and its properties were explored by Vist and Davis [130] in their study of the
DPPC/Chol phase diagram, which was constructed using data gathered from a variety of techniques including ${ }^{2} \mathrm{H}$ NMR. They found that for Chol concentrations greater than approximately $30 \mathrm{~mol} \%$ the membrane was in the $\mathrm{L}_{o}$ phase, and the deuterium NMR spectrum of DPPC- $\mathrm{d}_{62} /$ Chol was nearly invariant as a function of temperature from $30{ }^{\circ} \mathrm{C}$ to $50{ }^{\circ} \mathrm{C}$. The width of the DPPC $-\mathrm{d}_{62} /$ Chol spectrum was nearly double that found in the absence of cholesterol (i.e. in the $\mathrm{L}_{\mathrm{d}}$ phase), and reflected the presence of the highly ordered chains. In order to be absolutely sure that an $\mathrm{L}_{o}$ phase exists, it is imperative to have a carefully determined phase diagram. However, even in the absence of a phase diagram, the observation of substantial Cholinduced increases in chain order parameters may be taken as evidence of the possibility that an $\mathrm{L}_{\mathrm{o}}$ phase exists. Using published lipid/sterol phase diagrams together with measurements of lateral diffusion, the Lindblom group has characterized lateral diffusion in liquid-ordered and liquid-disordered phases on the basis of PFG NMR [153]. Results showed that in the $\mathrm{L}_{\mathrm{o}}$ phase for a given temperature, diffusion was reduced by a factor of two compared with the $\mathrm{L}_{\mathrm{d}}$ phase, and the corresponding activation energy associated with this diffusion increased substantially.

\subsection{Exploring the triangle: $\mathrm{SM} / \mathrm{PC} / \mathrm{Chol}$}

\subsubsection{The corners}

Apart from cholesterol, which does not on its own form bilayers in water, the pure lipid 'corners' of the ternary phase diagram provide a useful starting point for understanding the raft mixture, and for addressing the question of what phases these lipids assume.

7.2.1.1. SM. The ${ }^{31}$ P NMR spectra of brain SM membranes narrow as the temperature is increased through the gel to liquid crystalline $\left(\mathrm{L}_{\beta}\right.$ to $\left.\mathrm{L}_{\mathrm{d}}\right)$ phase transition. This transition is centred at approximately $35{ }^{\circ} \mathrm{C}$ [154] and is broad because of the distribution of chains found in this natural lipid. Filippov et al. [153] measured the diffusion coefficient $D_{L}$ of egg SM at $40{ }^{\circ} \mathrm{C}-60{ }^{\circ} \mathrm{C}$ in oriented membranes using PFG NMR. Apart from a reduction in $\mathrm{D}_{\mathrm{L}}$ at temperatures near $40{ }^{\circ} \mathrm{C}$, which was attributed to incomplete melting of the SM, the diffusion was characteristic of the $L_{d}$ phase (note that lateral diffusion in the gel $\left[\mathrm{L}_{\beta}\right]$ phase is too slow to be measured with PFG NMR). The activation energy characterizing the process of SM self-diffusion was $39 \mathrm{~kJ} / \mathrm{mol}$. Synthetic deuterium-labelled SM has recently become commercially available, allowing this lipid to be analyzed by ${ }^{2} \mathrm{H}$ NMR. Palmitoyl chain perdeuterated PSM is substantially analogous to DPPC bearing a single perdeuterated chain, and has an abrupt solid-ordered to liquiddisordered transition near $40{ }^{\circ} \mathrm{C}$. The latter result was determined using ${ }^{2} \mathrm{H}$ NMR by measuring the spectral width as a function of temperature [155]. Thus, the SM corner of the phase diagram is in

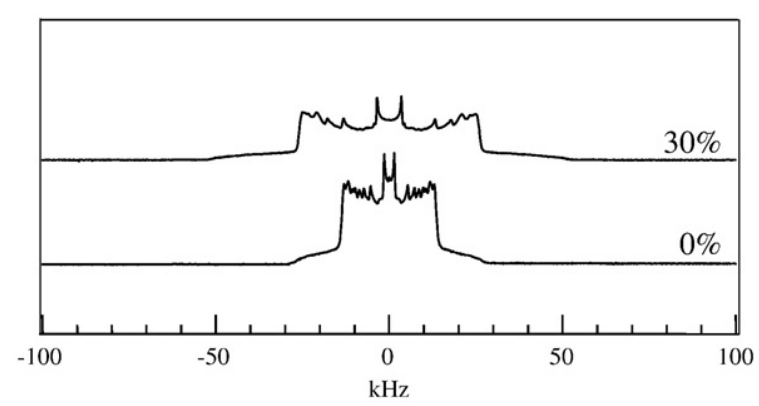

Fig. 6. The ${ }^{2} \mathrm{H}$ NMR spectrum of $s n-1$ chain-perdeuterated DPPC in the presence or absence of $30 \mathrm{~mol} \%$ ergosterol, at $T=45^{\circ} \mathrm{C}$. Both spectra show the Pake doublets that are characteristic of the axially symmetric chain motion that occurs in a liquid crystalline membrane. In the absence of sterol, i.e. in the $\mathrm{L}_{\mathrm{d}}$ phase, the spectrum is much narrower than in the presence of $30 \mathrm{~mol} \%$ sterol, in which case the membrane is in the $\mathrm{L}_{\mathrm{o}}$ phase. This figure is adapted from Hsueh et al. [151]. 
either the solid-ordered (gel) phase or liquid-disordered $\left(L_{d}\right)$ phase, depending on temperature and on the source of SM.

7.2.1.2. POPC or DOPC. POPC has been studied extensively by ${ }^{2} \mathrm{H} N M R$, using POPC with a perdeuterated palmitoyl chain. Aqueous multilamellar dispersions of POPC- $\mathrm{d}_{31}$ melt abruptly from a $\mathrm{L}_{\beta}$ phase to a $\mathrm{L}_{\mathrm{d}}$ phase at $-10^{\circ} \mathrm{C}[156]$. Thus, this corner of the ternary phase diagram is typically $\mathrm{L}_{\mathrm{d}}$.

Phosphatidylcholine species having two unsaturated chains, such as DOPC, have not been studied extensively using NMR; ${ }^{2} \mathrm{H}$ NMR studies have most commonly focused on lipids having saturated labelled chains, with the notable exceptions of Seelig and Seelig [157] and Oldfield et al. [158]. Interpretation of unsaturated chain order parameters is less straightforward than that of saturated chains, since the observed quadrupolar splittings are influenced not only by dynamic chain order but also by the permanent kink associated with the cis double bond. Recently, Warchawski and Devaux [159] used ${ }^{13} \mathrm{C}$ and ${ }^{1} \mathrm{H}$ NMR magnetization transfer NMR techniques to measure order parameters from C9 to C18 in DOPC. These order parameters were low and decreased significantly when the membrane was heated from $23{ }^{\circ} \mathrm{C}$ to $37{ }^{\circ} \mathrm{C}$. By measuring the lateral diffusion of DOPC as a function of temperature from $25^{\circ} \mathrm{C}$ to $60^{\circ} \mathrm{C}$ using PFG NMR, Filippov et al. [153] found that DOPC displayed temperaturedependent diffusion corresponding to an activation energy of $27 \mathrm{~kJ} / \mathrm{mol}$, which is characteristic of a loosely packed $\mathrm{L}_{\mathrm{d}}$ phase. Taken together, these data indicate that it is safe to conclude that the DOPC corner of the ternary phase diagram is in the $L_{d}$ phase at typical experimental temperatures, which are generally well above the DOPC main transition temperature of $-18{ }^{\circ} \mathrm{C}$.

\subsubsection{The edges}

7.2.2.1. SM/Chol. The solubility of cholesterol in bovine brain SM is approximately $50 \mathrm{~mol} \%$ [160]. The Epand group has shown that both oleoyl SM [161] and egg SM [128] can incorporate up to $60 \mathrm{~mol} \%$ Chol without any resultant formation of crystallites of Chol monohydrate. Guo et al. [160] interpreted ${ }^{13} \mathrm{C}$ NMR chemical shifts for BSM and suggested that at less than $50 \mathrm{~mol} \%$ Chol content, the action of Chol on SM was in some ways similar to its effect on DPPC. Chol, when present at sufficiently high concentrations, creates a $\mathrm{L}_{\mathrm{o}}$ phase, and at intermediate concentrations, allows the coexistence of $\mathrm{L}_{\mathrm{o}}$ and $\mathrm{L}_{\mathrm{d}}$ phases (for $T>T_{\mathrm{m}}$ ). Filippov et al. [153] determined the phase diagram for egg SM/Chol above the SM gel to $\mathrm{L}_{\mathrm{d}}$ phase transition temperature by using PFG NMR to measure temperature-dependent and Chol concentration-dependent changes in lateral diffusion. For Chol concentrations of approximately $5 \mathrm{~mol} \%$ or less, SM was in the $\mathrm{L}_{\mathrm{d}}$ phase and exhibited rapid lateral diffusion. At Chol concentrations between $\sim 5 \mathrm{~mol} \%$ and $20 \mathrm{~mol} \%$, SM diffusion showed a strong dependence on Chol concentration. However, only a single exponential was needed to fit this data, indicating that the SM was in 'fast exchange' between the $\mathrm{L}_{\mathrm{o}}$ and $\mathrm{L}_{\mathrm{d}}$ domains; therefore, the measured $\mathrm{D}_{\mathrm{L}}$ was the average value obtained over the non-homogeneous membrane. Finally, at Chol concentrations of $22 \mathrm{~mol} \%$ or more (the $\mathrm{L}_{\mathrm{o}}$ phase), the diffusion of SM was slowed by a factor of two compared to diffusion in the $\mathrm{L}_{\mathrm{d}}$ phase, and the measured diffusion was nearly independent of cholesterol content. Thus, the SM/Chol 'edge' of the $\mathrm{SM} / \mathrm{PC} / \mathrm{Chol}$ triangle should contain, for temperatures above the $T_{\mathrm{m}}$ for the particular SM being studied, a region of $\mathrm{L}_{\mathrm{d}} / \mathrm{L}_{\mathrm{o}}$ phase coexistence at cholesterol concentrations between approximately $5 \mathrm{~mol} \%$ and $20 \mathrm{~mol}$ $\%$, and an $\mathrm{L}_{\mathrm{o}}$ phase at Chol concentrations between approximately $20 \mathrm{~mol} \%$ and $50 \mathrm{~mol} \%$. Bunge et al. [155] measured the temperature dependence of the ${ }^{2} \mathrm{H}$ NMR spectral width for perdeuterated PSM membranes containing $40 \mathrm{~mol} \% \mathrm{Chol}$. The observed lack of a sharp change in spectral width implies that these membranes are in the $\mathrm{L}_{0}$ phase. As well, at $40{ }^{\circ} \mathrm{C}$ these membranes had 'plateau' chain order parameters greater than 0.40 , consistent with the $\mathrm{L}_{o}$ phase. (The 'plateau' is a region of nearly constant chain order parameter arising from the section of the hydrocarbon chain near the membrane/water interface). Note that the maximum ${ }^{2} \mathrm{H}$ NMR chain order parameter is 0.50 , which would result from an all-trans chain undergoing axial rotation. The hydrocarbon chains of membranes in the $\mathrm{L}_{o}$ phase approach this level of organization except for the methylenes and methyl group near the bilayer centre.

At temperatures below the SM $T_{\mathrm{m}}$, the $\mathrm{L}_{\mathrm{d}}$ phase would presumably be replaced by the solid-ordered (gel) phase, giving rise to regions of pure $\mathrm{L}_{\beta}$ (below $\sim 5 \mathrm{~mol} \% \mathrm{Chol}$ ) and coexisting $\mathrm{L}_{\beta} / \mathrm{L}_{\mathrm{o}}$ (from $\sim 5$ to $20 \mathrm{~mol} \%$ ). Since the $\mathrm{SM} / \mathrm{PC} / \mathrm{Chol}$ phase diagram has usually been determined at room temperature, the $\mathrm{L}_{\beta}$ phase is typically observed, but this phase is probably not important in cell membranes (except perhaps in cells undergoing apoptosis). Recently, the headgroup of egg SM was monitored by ${ }^{31} \mathrm{P}$ NMR [162] and cholesterol was shown to modify its packing/dynamics below $T_{\mathrm{m}}$. Aussenac et al. [154] measured the spectrum of $33 \mathrm{~mol} \%$ deuteriumlabelled Chol in BSM at $T<T_{\mathrm{m}}$ and observed some reduction in Chol mobility at $20{ }^{\circ} \mathrm{C}$ and nearly complete immobilization, consistent with Chol solid phase/crystal formation, at $5{ }^{\circ} \mathrm{C}$. This finding implies that the inaccessible Chol triangle at the top of the phase diagram increases in size as temperature is lowered. Keeping Chol concentrations below $50 \mathrm{~mol} \%$ and the temperature no lower than room temperature is thus advisable for anyone seeking to tame this phase diagram!

7.2.2.2. SM/unsaturated PC. POPC/SM binary mixtures have not been studied extensively by NMR as yet. A membrane composed of a 1:1 mixture of POPC and PSM exhibited $\mathrm{L}_{d}$ spectra at $40{ }^{\circ} \mathrm{C}$, with no evidence of phase coexistence despite the fact that perdeuterated PSM had a larger order parameter than POPC-d $\mathrm{d}_{31}$ [155].

Filippov et al. [163] measured the lateral diffusion of membranes composed of a 1:1 mixture of DOPC and egg SM as a function of temperature, and found that from $30{ }^{\circ} \mathrm{C}$ to $60{ }^{\circ} \mathrm{C}$, the diffusion coefficient $D_{\mathrm{L}}$ increased from $7.5 \times 10^{-12} \mathrm{~m}^{2} / \mathrm{s}$ to $22 \times 10^{-12} \mathrm{~m}^{2} / \mathrm{s}$. At each temperature tested, the mixed membrane's diffusion coefficient was slower than the $\mathrm{D}_{\mathrm{L}}$ determined for DOPC and faster than that determined for SM. The diffusion was monoexponential in nature, so as a result, the membrane was not in a mixed $L_{d} / L_{o}$ state. However, since the PFG method cannot detect gel-phase lipids because their diffusion is too slow, the authors pointed out that gel 'patches' may exist in this mixture at temperatures below $40{ }^{\circ} \mathrm{C}$, which is the transition temperature of egg SM.

For phase diagrams with either DOPC or POPC as the low-melting lipid, along the $\mathrm{PC} / \mathrm{SM}$ edge of the raft triangle, the prevalence of the $\mathrm{L}_{\beta}$ (gel) phase will increase as one approaches the SM corner for temperatures below the main transition temperature of SM, approximately $40{ }^{\circ} \mathrm{C}$. (The precise temperature will depend on the type of SM being studied.) The concentration of SM at which the gel phase is first observed at a given temperature has not been examined in detail by NMR. Above the main transition temperature, this entire edge of the triangle will be in the $\mathrm{L}_{\mathrm{d}}$ phase.

7.2.2.3. Unsaturated $P C / C h o l$. In the liquid crystalline phase, POPC- $d_{31}$ order parameters increase as Chol is added to the membrane, reaching typical $\mathrm{L}_{\mathrm{o}}$ values at a Chol concentration of approximately $30 \mathrm{~mol} \%$ [164]. The POPC- $\mathrm{d}_{31} /$ Chol partial phase diagram was first determined by ${ }^{2} \mathrm{H}$ NMR by Thewalt and Bloom [165]. POPC- $\mathrm{d}_{31} /$ Chol membranes have a well-defined melting transition temperature that is independent of Chol concentration between approximately 10 and $25 \mathrm{~mol} \%$ Chol. This three-phase line is similar to that observed by Vist and Davis [130] for DPPC/Chol (although more than $40{ }^{\circ} \mathrm{C}$ lower in temperature) and according to Gibbs, its presence means that POPC$\mathrm{d}_{31} /$ Chol will exhibit $\mathrm{L}_{\mathrm{o}} / \mathrm{L}_{\mathrm{d}}$ phase coexistence at temperatures above $T_{\mathrm{m}}$. Micron-sized $\mathrm{L}_{\mathrm{o}}$ and $\mathrm{L}_{\mathrm{d}}$ domains would be evident as superimposed deuterium NMR spectra reflecting very limited exchange of 
lipids between the different phases. Such domains are not observed, but spectra do show broadened lines which are evidence of membrane heterogeneity [166]. The upper limit of temperature at which $\mathrm{L}_{\mathrm{o}} / \mathrm{L}_{\mathrm{d}}$ domains coexist is also being studied by these authors. At temperatures of physiological interest, the POPC/Chol edge of the phase triangle will be either featureless (at temperatures above $L_{d} / L_{o}$ coexistence) or will have a region of $\mathrm{L}_{\mathrm{d}} / \mathrm{L}_{\mathrm{o}}$ coexistence at intermediate sterol concentrations.

Warschawski and Devaux [159] have reported that Chol exerts a substantial ordering effect on DOPC. The order parameters from the 'lower' half of the oleoyl chains (with the exception of the methyl group) increased very significantly upon the addition of $33 \mathrm{~mol} \%$ Chol. The authors propose that Chol does induce a type of liquidordered phase in DOPC [167], but point out that Chol may be affecting the average orientation of the unsaturated 'kink' as well as the degree of conformational ordering. Thus, it is not clear whether the ordering observed is entirely analogous to that seen in the 'standard' $\mathrm{L}_{\mathrm{o}}$ phase. Filippov et al. [153] found that DOPC/Chol was in the $\mathrm{L}_{d}$ phase at temperatures ranging from $25{ }^{\circ} \mathrm{C}$ to $50{ }^{\circ} \mathrm{C}$ and for Chol concentrations of up to $47 \mathrm{~mol} \%$. There was no sign of a region of increased dependence of the diffusion coefficient on Chol concentration, as was seen for SM/Chol. However, the measured diffusion coefficients did depend on Chol concentration, and the activation energy associated with the lateral diffusion of DOPC increased slightly with increasing Chol. On balance, the evidence seems to favour the absence of $\mathrm{L}_{\mathrm{d}} / \mathrm{L}_{o}$ phase coexistence for DOPC/ Chol. Therefore, at all typical experimental temperatures, this 'edge' of the triangle will be in the $L_{d}$ phase until the solubility limit for Chol in DOPC is reached.

\subsubsection{The middle}

We can now venture into the middle of the triangle. There are few NMR investigations of the ternary systems, either POPC/SM/Chol or DOPC/SM/Chol. For membranes composed of equimolar amounts of POPC, BSM and cholesterol, Aussenac et al. [154] concluded that Chol was able to rapidly exchange between environments rich in POPC or $\mathrm{SM}$, respectively. No evidence of micron-sized phase domains was observed. Recently, Bunge et al. [155] concluded that at $40{ }^{\circ} \mathrm{C} \mathrm{POPC/}$ PSM/Chol (37.5:37.5:25 mol ratio) was also heterogeneous on a scale of at least $45 \mathrm{~nm}$. Again, the absence of superimposed $\mathrm{L}_{\mathrm{o}}$ and $\mathrm{L}_{\mathrm{d}}$ spectra means that this ternary phase diagram is still quite wild from an NMR standpoint.

Turning to the DOPC/SM/Chol triangle, we step up towards the cholesterol vertex from the middle of the DOPC/SM side. Filippov et al. [163] have determined lipid lateral diffusion coefficients for oriented stacked bilayers of DOPC/SM/Chol for temperatures between $20{ }^{\circ} \mathrm{C}$ and $60{ }^{\circ} \mathrm{C}$. In this study, the ratio of the phospholipids was held at unity, and the proportion of cholesterol was varied up to $45 \mathrm{~mol} \%$. In contrast to the binary mixture of $\mathrm{SM} / \mathrm{Chol}$ described above, the diffusion data clearly showed two components-one fast and one slow-for intermediate Chol concentrations at certain temperatures. The $D_{L}$ value for the fast component was similar to that observed for DOPC/Chol, and $D_{L}$ for the slow component was similar to $D_{L}$ in $S M /$ Chol. The authors concluded that the $\mathrm{L}_{d}$ phase was enriched in DOPC and the $\mathrm{L}_{o}$ phase was enriched in $\mathrm{SM}$, and that the lipids were not exchanging quickly between the phases. This two-phase region was apparent for cholesterol concentrations from $7.5 \%$ to $33 \%$, between $T=34^{\circ} \mathrm{C}$ and $T=55^{\circ} \mathrm{C}$. However, at higher temperatures, the minimum cholesterol content required to observe the two phases was increased. At $60{ }^{\circ} \mathrm{C}$ the membrane exhibited a single diffusion coefficient characteristic of a homogeneous liquid crystalline phase; this $\mathrm{D}_{\mathrm{L}}$ value was close to the average of the individual $D_{L}$ values for DOPC/ Chol and SM/Chol. At $30^{\circ} \mathrm{C}$, the phases encountered along the path up the centre of the ternary triangle are dependent on Chol concentration: At low Chol concentrations, the centre contains gel and $\mathrm{L}_{\mathrm{d}}$ phases; for Chol concentrations between $22 \%$ and 33\%, the centre contains $\mathrm{L}_{\mathrm{o}}$ and $\mathrm{L}_{\mathrm{d}}$ phases; and for high Chol concentrations, the centre contains the $\mathrm{L}_{\mathrm{o}}$ phase. At temperatures just above the $T_{\mathrm{m}}$ of SM, the gel phase will not be present and the $\mathrm{L}_{\mathrm{o}} / \mathrm{L}_{\mathrm{d}}$ coexistence will commence at a lower Chol concentration. At $50{ }^{\circ} \mathrm{C}$ the zone of $\mathrm{L}_{\mathrm{o}} / \mathrm{L}_{\mathrm{d}}$ coexistence ranges from $15 \%$ to $33 \% \mathrm{Chol}$, while at $60{ }^{\circ} \mathrm{C}$, a single liquid crystalline phase is present for all Chol concentrations.

DOPC evidently encourages $\mathrm{L}_{o} / \mathrm{L}_{d}$ phase separation in SM/Chol to a greater extent than does POPC. Some insight into this phenomenon may be gained from the recent work by Soni et al. [135], who measured the interactions between palmitoyl-SM/cholesterol and either POPE or 1-palmitoyl, 2-docosahexaenoyl PE (PDPE) using ${ }^{2} \mathrm{H}$ NMR (palmitoyl chains of PE and SM labelled in turn). The multiply unsaturated PDPE experienced a much smaller increase in chain order upon the incorporation of cholesterol into PE/SM 1:1 membranes than did the mono-unsaturated POPE. Increased lipid chain unsaturation reduces the affinity for cholesterol and enhances the difference between $\mathrm{L}_{\mathrm{o}}$ and $\mathrm{L}_{\mathrm{d}}$ domains.

Notably, NMR studies on ternary 'model raft' membranes have often used DPPC as the 'high-melting' saturated lipid, in place of SM (see, for example, [64]). At some temperatures and compositions, ${ }^{2} \mathrm{H}$ NMR analysis of these ternary mixtures indicates that labelled DPPC molecules in $\mathrm{L}_{\mathrm{o}}$ and $\mathrm{L}_{\mathrm{d}}$ domains are not in 'fast exchange'. The spectra of the $L_{o}$ and $L_{d}$ phases appear superimposed, demonstrating that large (i.e. micron-sized) coexisting domains of the two phases are present. Orädd et al. [168] used PFG NMR combined with completely deuterium-labelled DPPC- $\mathrm{d}_{75}$ and C25 fluorine-labelled Chol to examine the phase behaviour of DOPC/DPPC/Chol, as determined by lipid-specific lateral diffusion measurements. The DOPC/DPPC ratio was unity and the Chol content was either $15 \mathrm{~mol} \%$ or $30 \mathrm{~mol} \%$. At high temperatures, all three membrane constituents had the same diffusion coefficient, indicating a well-mixed, single phase environment. At lower temperatures $\left(30{ }^{\circ} \mathrm{C}\right.$ or below), the lipids separated into slow- and fast-diffusing populations, as was described above for the DOPC/SM/Chol membrane. Because of the isotopic labelling, however, it was possible to determine that DPPC displayed the slow component exclusively. DOPC and Chol exhibited both slow and fast components, although the majority of DOPC diffused rapidly. For the $15 \%$ Chol system, the amount of the slow-diffusing component was always very small, implying that there was only a very low proportion of $\mathrm{L}_{\mathrm{o}}$ phase. Polozov and Gawrisch [169] were able to distinguish $\mathrm{L}_{\mathrm{o}}, \mathrm{L}_{\mathrm{d}}$ and $\mathrm{L}_{\beta}$ phases in mixtures of DOPC/DPPC/Cholesterol using magic angle spinning ${ }^{1} \mathrm{H}$ NMR, a technique which shows promise for the study of domains in cell membranes.

NMR determinations of ternary lipid phase diagrams can also reveal tie lines and regions of three-phase coexistence [170]. However this has not yet been done for the model raft system containing SM.

\section{Spin-label ESR}

Electron spin resonance (ESR) spectroscopy of spin-labelled lipid chains is used to determine phase diagrams for sphingolipidglycerolipid-cholesterol mixtures by assessing sensitivity to molecular mobility and orientational order in the membrane. With suitable choice of the position of chain labelling, $\mathrm{L}_{\beta}, \mathrm{L}_{\mathrm{o}}$ and $\mathrm{L}_{\mathrm{d}}$, (or $\mathrm{L}_{\alpha}$ ) phases can be resolved in two-component spectra (see Fig. 7). Spectra from gel phases are broadest and contain the largest range of spectral hyperfine splittings; those from $\mathrm{L}_{0}$ phases are narrowest, with an intermediate range of hyperfine splittings; and those from $L_{d}$ phases are narrow, with the smallest range of hyperfine splittings. The fractional populations of spin label in the coexisting phases can be determined by spectral subtraction and/or addition (see, for example, $[171,172])$.

The distribution of the spin-labelled lipid between the coexisting phases is determined by the usual lever rule (see Section 2.1), taking into account possible differences in partitioning of the spin-labelled probe between the two coexisting phases. For example, in a binary 

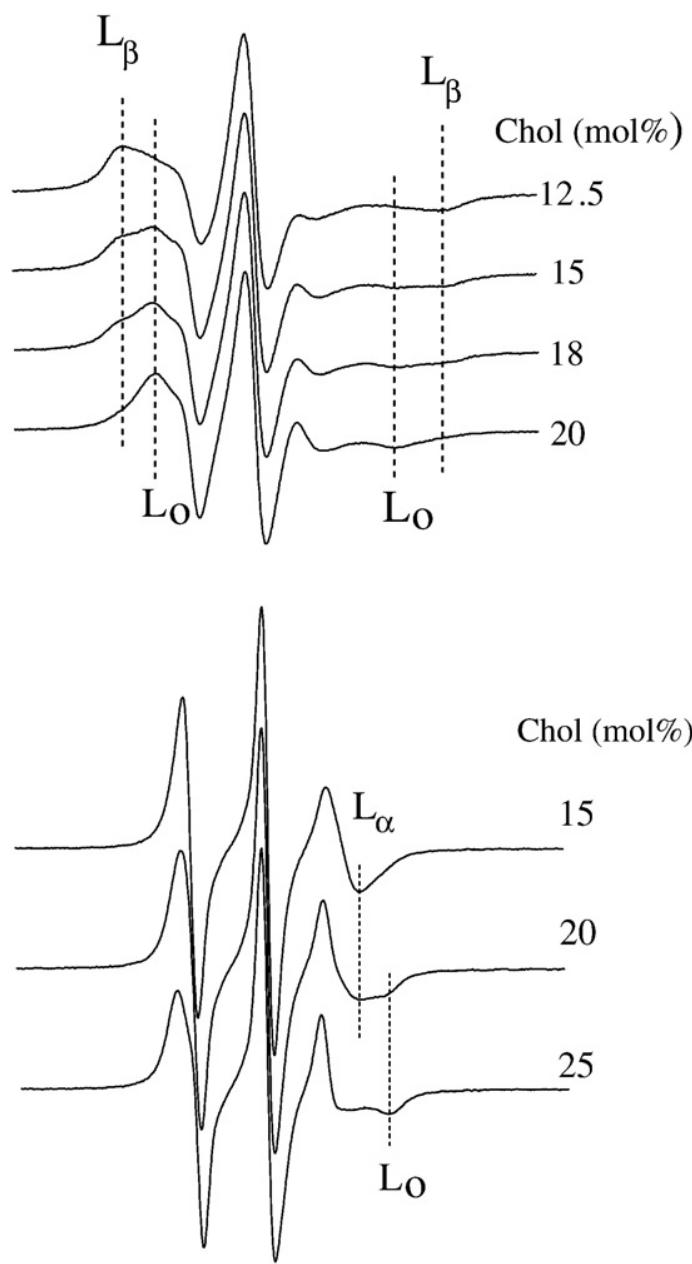

Fig. 7. Two-component ESR spectra indicating phase coexistence: ${ }^{14} \mathrm{C}$ atom spinlabelled lipid chains $(0.5 \mathrm{~mol} \%)$ in $\mathrm{N}$-palmitoyl sphingomyelin/cholesterol mixed membranes. (Top panel) Membranes with the indicated cholesterol contents, spanning gel-liquid ordered $\left(\mathrm{L}_{\beta}-\mathrm{L}_{0}\right)$ phase separation, were analyzed at $22{ }^{\circ} \mathrm{C}$. (Bottom panel) Membranes with the indicated cholesterol contents, spanning liquid ordered-liquid disordered $\left(\mathrm{L}_{0}-\mathrm{L}_{\alpha}\right)$ phase separation, were analyzed at $50{ }^{\circ} \mathrm{C}[171]$.

lipid-cholesterol mixture, the fraction $(f)$ of spin label in the fluid phase, $F$, depends on the mole fraction $(X)$ of cholesterol according to:

$f=\frac{K_{\mathrm{p}}\left(X-X_{S}\right)}{X_{F}-X_{S}+\left(K_{\mathrm{p}}-1\right)\left(X-X_{S}\right)}$

where $X_{F}$ is the mole fraction of cholesterol in phase $F$, and $X_{S}$ is the mole fraction in the more 'solid-like' phase, $S$. The partition coefficient of the spin-labelled lipid into the $F$-phase, relative to the $S$-phase, (for equal mole fractions of both) is $K_{\mathrm{p}}$ (see Eq. (1)). For $K_{\mathrm{p}}=1$ (i.e. a situation in which there is no differential partitioning of the spinlabelled lipid between the two phases), Eq. (2) reduces to the familiar lever rule: $f=\left(X-X_{S}\right) /\left(X_{F}-X_{S}\right)$. Eq. (2) forms the basis for determining the phase boundaries, which are defined by $X_{F}$ and $X_{S}$. A series of cholesterol concentrations, $X$, are required to allow for differential partitioning of the spin label (i.e., $K_{\mathrm{P}} \neq 1$ ), in order to determine the tieline at a given temperature in a binary mixture. For the spin label used in Fig. 7, it was found that $K_{\mathrm{P}} \sim 1$ for the $\mathrm{L}_{\beta}-\mathrm{L}_{\mathrm{o}}$ coexistence region [171], and $K_{\mathrm{P}} \sim 1$ also for a spin-labelled phosphatidylcholine in the $\mathrm{L}_{\beta}-\mathrm{L}_{\alpha}$ coexistence region of DPPC/dilauroyl PC/Chol mixtures [172,173]. Fig. $8 \mathrm{~A}$ shows the temperature-composition phase diagram that was determined using the above method for the $\mathrm{L}_{\beta}-\mathrm{L}_{0}$ coexistence region in binary mixtures of PSM and Chol. Because this is a binary mixture, the tie-lines are horizontal, corresponding to constant temperature.
Determination of the thermodynamic tie-lines in ternary mixtures is always more difficult than for binary mixtures because of the added complexity introduced by the third component. Nevertheless, it has been shown that the principles illustrated above are sufficient to determine tie-lines in a ternary system, although analysis is constrained by the following: Along a tie line, (1) the ESR spectrum is a linear superposition of the spectra from the two phases and (2) $K_{\mathrm{P}}$ is constant. Working within these constraints, Chiang et al. [173] carried out the combined analysis of over 100 spin-labelled samples of different lipid composition and demonstrated the feasibility of this spin-label method. The authors were able to determine both the phase boundaries and the tie-lines over the full range of $\mathrm{L}_{\beta}-\mathrm{L}_{\alpha}$ phase coexistence in the ternary phase diagram of DPPC/DLPC/Chol membranes at $24{ }^{\circ} \mathrm{C}$ (see Fig. 8B). A weighted least-squares analysis was required to identify the tie-lines, and-even when not visually obvious-two-component spectra were demonstrated by isosbestic points in the absorption-display ESR spectra of samples lying along a tie-line [172].

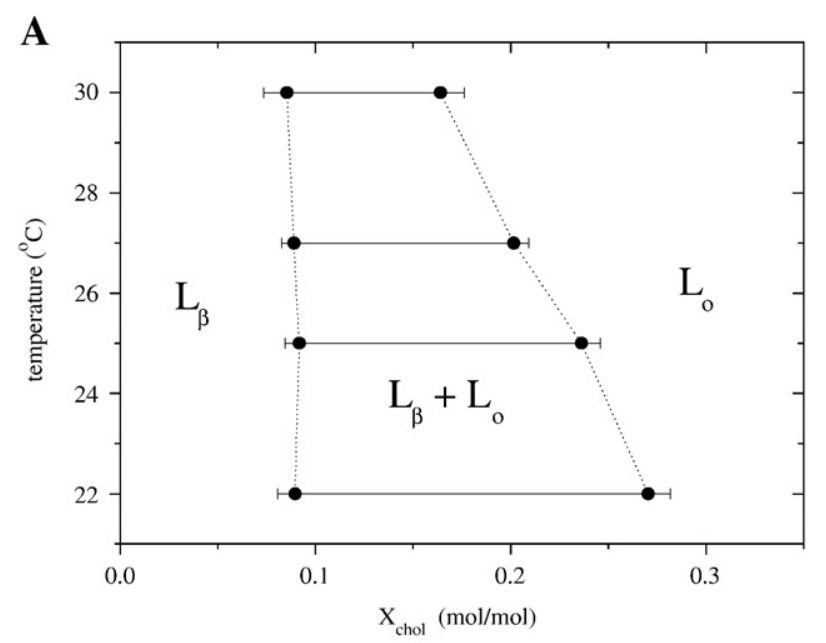

B

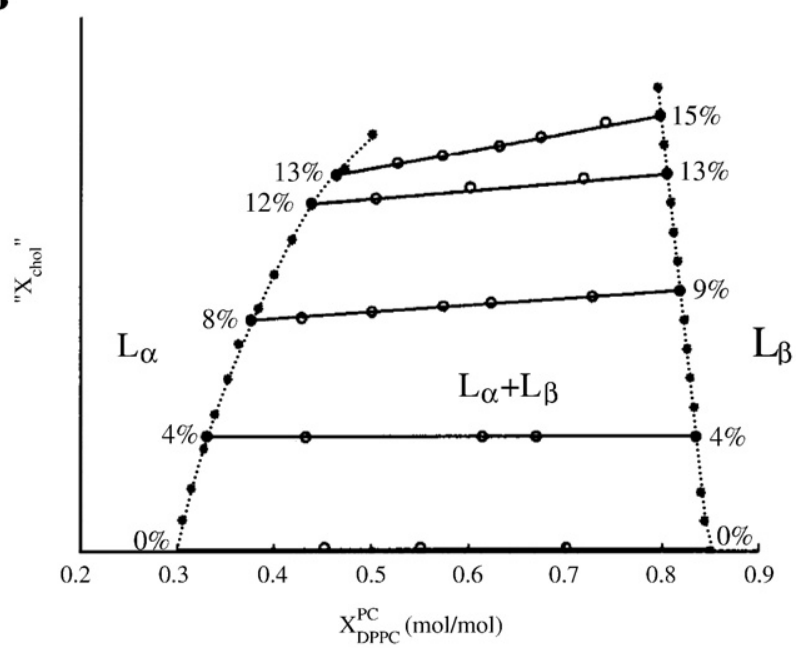

Fig. 8. Phase boundaries in lipid-cholesterol mixtures determined by spin-label ESR. Phase boundaries are indicated by dotted lines; solid lines are tie-lines. (A) Temperature-composition binary phase diagram: gel-liquid ordered $\left(\mathrm{L}_{\beta}-\mathrm{L}_{\mathrm{o}}\right)$ phase boundaries in PSM/Chol binary mixtures. (Data from [171]) (B) Constant-temperature ternary phase diagram: Gel-liquid crystalline $\left(\mathrm{L}_{\beta}-\mathrm{L}_{\alpha}\right)$ phase boundaries in DPPC)/ dilauroyl $\mathrm{PC} / \mathrm{Chol}$ ternary mixtures at $24^{\circ} \mathrm{C}$. The $x$-axis $\left(X_{\mathrm{DPPC}}^{\mathrm{PC}}\right)$ is the mole fraction of DPPC, relative to total PC (DPPC+dilauroyl PC). The $y$-axis represents the mole fraction of Chol, relative to total PC. Actual mole percentages of $\mathrm{Chol}\left(X_{\text {chol }}\right)$ are indicated for the points at the ends of the tie lines. (Adapted from [173]). 
The tie-lines shown in Fig. 8B for DPPC/DLPC/Chol membranes are not all horizontal, but are tilted relative to the DLPC-DPPC axis at the higher Chol concentrations. This illustrates the well-established principle that it is essential to know the orientation of the tie lines in order to define the compositions of the coexisting phases in ternary mixtures. It is not sufficient simply to know the position of the phase boundaries. Fluorescence microscopy, for instance, is capable (in principle) of visualizing the coexisting phases but does not provide thermodynamic information on the composition of the phases.

The ability to determine tie-lines is, therefore, a crucial aspect of the ESR spectroscopic visualization of co-existing phases such as those illustrated in Fig. 8. There are currently no exhaustive studies of SMcontaining ternary mixtures using this method. Nevertheless, evidence for $\mathrm{L}_{\beta}-\mathrm{L}_{o}$ phase coexistence in sphingolipid/glycerolipid/Chol mixtures has been obtained from the two-component ESR spectra of spinlabelled PC and SM using lipid species of natural origin [174]. The twocomponent ESR spectra of spin-labelled phosphatidylcholine have been used to demonstrate $L_{\beta}-L_{d}$ phase coexistence in SM/PC mixtures [175], and $\mathrm{L}_{\beta}-\mathrm{L}_{o}$ phase coexistence in $\mathrm{SM} /$ Chol mixtures [141].

Before going on to discuss the implications of two-component ESR spectra in the wider context of phase separation as examined by other techniques, it is useful to briefly survey earlier spin-label ESR studies that have provided evidence for phase separation in Chol-containing membranes. These studies, which originated with the pioneering work of Shimshick and McConnell [2] on the partitioning of the small spin-label TEMPO (2,2,6,6-tetramethylpiperidine-1-oxyl) into membranes of mixed lipids [2], rely mostly on discontinuities in temperature or in the composition dependence of the spectral parameters to establish phase boundaries. In particular, spin-labelled phospholipid headgroups have been used in early studies of PC/Chol mixtures that analyzed the dependence of the ESR spectra on Chol content $[176,177]$. Subsequently, Sankaram and Thompson [104] used discontinuities in the composition dependence of spectral splittings from spin-labelled phospholipid chains (at constant temperature) to empirically estimate the boundaries of $\mathrm{L}_{\alpha}-\mathrm{L}_{0}$ phase coexistence for binary mixtures of cholesterol with DPPC or with BSM. Further substantiation of the $\mathrm{L}_{\alpha}-\mathrm{L}_{\mathrm{o}}$ phase boundaries for DPPC/Chol mixtures was obtained from the two-component ESR spectra of a phospholipid biradical in which both chains were spin-labelled, giving rise to highly characteristic lineshapes for the $\mathrm{L}_{\mathrm{o}}$ phase [178].

\section{The uncertainties}

\subsection{Monolayers vs. bilayers}

Interestingly, the domains observed in monolayers and in GUVs exhibit differing behaviours. In monolayers, there is more variety in the sizes of domains, which rarely coalesce unless the system is close to a critical point or unless the domains are forced into close contact by electric field gradients [179]. In GUVs, particularly those with ternary mixtures displaying liquid immiscibility (e.g. DOPC/SM/Chol), domains often collide and coalesce into one bright domain and one dark domain within a period of minutes. This behaviour, however, is not observed in GUVs displaying gel-fluid phase coexistence, or in GUVs composed of complex mixtures, such as pulmonary surfactants. Also, in GUVs, the distribution of the different lipids between the outer and inner leaflets is similar, and domains in each leaflet overlap and appear to be in register [56,60,78].

Given the differences described above, one might ask whether there is any general method for translating between the phase diagrams of monolayers and bilayers. One way to accomplish such a comparison has been proposed by Harden McConnell [79], and involves inverting the pressure axis into temperature to translate a monolayer phase diagram into a bilayer phase diagram. Although this approach yields a rough correlation between the monolayer and bilayer phase diagrams of a ternary mixture of DOPC/DPPC (1:1) with varying Chol, no absolute correlation is observed in the full phase diagrams between the miscibility boundary in the GUV bilayer and the transition between the $\alpha$ - and $\beta$-regions in the monolayer [79]. Additional comments concerning the correspondence between monolayer and bilayer phases in two-phase lipid systems have been provided by McConnell and Vrljic [70].

So, a key unresolved issue is the relative value of monolayers and GUVs for providing accurate insights into lipid behaviour and mixing in biomembranes. There is a tendency to assume that the bilayer structure of a GUV makes it the better model for understanding what goes on in biomembranes, because the hydrocarbon chains in each half of the GUV and biomembrane bilayer appose each other along a nonpolar interface rather than projecting into a gas phase (e.g., air, argon, or nitrogen). However, it is important to remember that GUVs, at least as they are currently made (although a protocol to generate asymmetric GUVs was reported [180]), do lack key features of biomembranes, such as the lipid compositional asymmetry that is known to exist in the inner and outer leaflets of biomembranes. This becomes particularly important when the coexisting liquid domains differ in thickness, as is the case when Chol induces two-phase coexistence by increasing hydrocarbon chain order [181]. The overlapping of domains observed within the apposing halves of PSM/ POPC/Chol GUVs would suggest that symmetric lipid compositional distributions across the bilayer may contribute favourably to transbilayer coupling of domains, and may affect the overall lateral miscibility behaviour. However, Merkel et al. [182] have demonstrated transbilayer coupling in asymmetric planar membranes, and their results show that it is clear that the domains observed in GUVs are large and can coalesce within minutes at room temperature to micron-size macrodomains. In contrast, the microdomains observed in monolayers are smaller and usually decrease in size as the surface pressure increases, ultimately becoming too small to be observed by epifluorescence microscopy at the high surface pressures thought to mimic packing conditions in biomembranes.

We are currently left to speculate about how the limitations of monolayers and GUVs affect what can be learned about microdomain existence and behaviour in biomembranes. Neither the monolayer nor GUV system, as presently configured, duplicates the small size of the microdomains ( $\leq 50 \mathrm{~nm}$ diameter) reported to exist in biomembranes $[12,183]$. However, the lipid combinations studied to date in monolayers and GUVs have been relatively simple compared to the complex mixtures of lipid species known to occur in many biomembranes, with the exception of the intriguing monolayer study by Keller et al. [83]. Moreover, the effects of integral and peripheral membrane proteins on lipid-mixing behaviours in the GUV and monolayer model systems have not been adequately addressed, and an examination of these effects must continue to be developed and pursued (e.g. [184]). Therefore, the 'jury is still out' for the monolayer and GUV model membrane systems. It should be noted that, in addition to monolayers and GUV, multilamellar vesicles (MLV) are commonly used in some phase studies (particularly in NMR spectroscopy) because they afford much greater signal-to-noise ratios. However, some of the differences between MLVs and GUVs originate in the arrangement of closely apposed bilayers, and this is especially relevant in the ability of domains within individual layers to bulge out of the plane of the membrane. This is often seen in GUVs, but such configurations are not favourable in MLVs. How much we can ultimately learn about microdomains and lipid miscibility in biomembranes by using these model membrane systems will depend upon our ability to effectively decipher data obtained with more complex combinations of lipids and proteins.

In this respect it is interesting to mention a recently reported study regarding liquid domain formation in asymmetric Montal-Mueller planar bilayers [185]. This model system allows controlling the composition of each leaflet of the lipid bilayer offering possibilities to simultaneously explore the vertical and lateral lipid crosstalk 
originated in an asymmetric membrane. For example, these authors report that when domains are present in asymmetric membranes, each leaflet contains regions of three distinct lipid compositions, implying strong interleaflet interactions, proposing that mechanisms of domain induction between the outer and inner leaflets of cell plasma membranes do not necessarily require the participation of membrane proteins [185].

\subsection{Microscopy vs. spectroscopy}

As previously discussed, the information used to construct phase diagrams may come from a variety of different sources, and it is known that the information derived from spectroscopic techniques differs from that obtained from microscopy, in which phase co-existence is detected by direct visualization of the lipid domains. Veatch and Keller [13] discussed these differences in the case of the PSM/POPC/Chol system, and Fig. 9A is taken from their work. A very similar system (BSM replacing PSM), was studied by Pokorny et al. [122], using the kinetics of carboxyfluorescein efflux induced by a peptide ( $\delta$-lysin) (Fig. 9C).

According to Pokorny et al. [122] the quantitative agreement is higher with the de Almeida et al. diagram [186] (Fig. 9B), in the way that phase separation is obtained in the left-hand region of the diagram. This was also the conclusion obtained from microscopic models for these ternary mixtures [187]. Further experimental evidence for these types of diagrams and their application to the distribution of proteins in lipid microdomains was reported by Nicolini et al. [188].

There is a certain amount of dissent in the literature regarding the construction and interpretation of microscopy-based phase diagram data. Disagreement is not surprising, since as stated above, microscopy at its current resolution limits cannot reveal the existence of very small domains, and each technique has its strengths and weaknesses. Some issues can be ruled out as having limited relevance, such as the discussion of the eventual curvature effects in the smaller LUV (large unilamellar vesicles) used in the spectroscopic approaches, as compared to the larger GUV of microscopy. This difference can be ruled out because LUV have no significant curvature stress and thus there is an identical lipid distribution between the two leaflets. Differences between the phase diagrams obtained by fluorescence microscopy (Fig. 9A) and by fluorescence spectroscopy (Fig. 9B) could be due to the use of quenchers in the latter procedure. However, no quencher methodologies were used in the determination of the lefthand side of the spectroscopic diagram (Fig. 9B), but only the variation of fluorescence and lifetimes of suitable anisotropy probes, which is important to note because this is one of the regions where there is a greater variance as compared to the microscopy diagram. Another point under discussion is if the nanoscopic domains obtained from spectroscopy (in case they are not transient density fluctuations) can be considered to be distinct phases. If this is not the case, the phase diagrams and particularly the tie-lines would have no meaning. Although in classic terms (according to Gibbs) phase separation would proceed to infinity, no critical restriction on size would prevent the phase concept from being applied to nanodomains, which in any case, correspond to at least a few thousand molecules. Although it is not clear why the system is not driven to infinite phase separation in order to avoid the unfavourable line tension, it could be that apart from other explanations such as the affinity of Chol for domain edges $[22,75]$, there is also a compensation from the concomitant increase in the entropy of the system. A discussion favouring the utilization of "spectroscopic phase diagrams" was recently presented [189].

Although the information from microscopy is invaluable in that it is not possible to obtain information about domain shapes from any other approach, there are also limitations. As we described in Section 3 , the accuracy of the phase diagrams obtained needs to be revisited using other experimental approaches such as fluorescence imaging analysis [50] and measurement of lifetimes, polarization, and spectral shifts of suitable probes [31-33,190].

When comparing ESR and fluorescence microscopy results, the major distinguishing feature of results from spin-label ESR is the latter's ability to demonstrate fluid-phase coexistence directly in binary mixtures of PC or SM with cholesterol $[171,178]$. Fluorescence microscopy, on the other hand, has been unable to detect such phase coexistence in binary mixtures with Chol $[24,78,140]$. It has been suggested that the large coexisting fluid domains visualized by fluorescence microscopy in GUVs composed of Chol-containing ternary lipid mixtures may be an artifact induced by high levels of

A
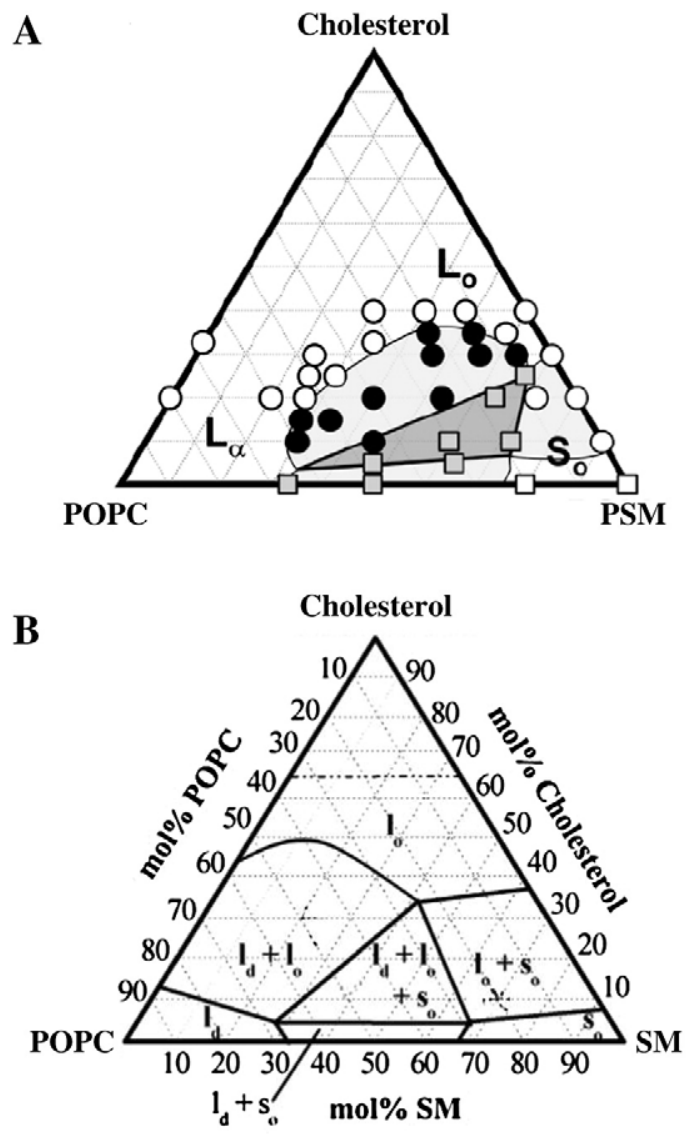

C

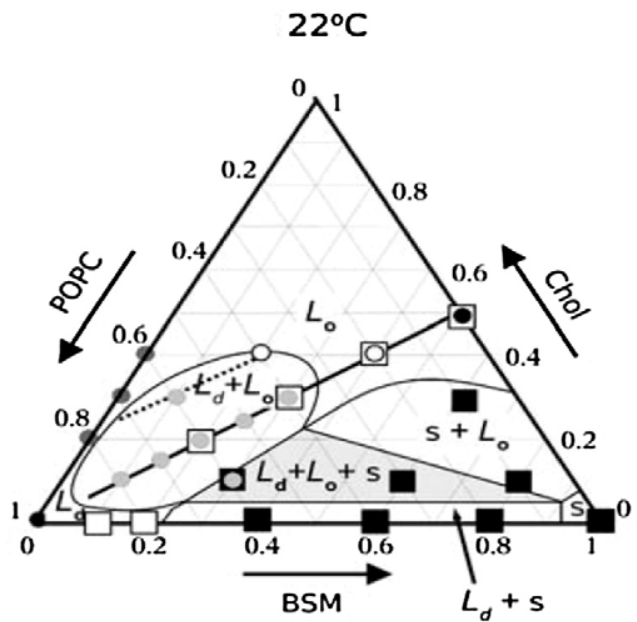

Fig. 9. Comparison of $\mathrm{PSM} / \mathrm{POPC} / \mathrm{Chol}$ phase diagrams obtained by: (A) optical microscopy [173], (B) fluorescence spectroscopy [186], (C) the kinetics of carboxyfluorescein efflux induced by a peptide ( $\delta$-lysin), [122]. The symbols reproduced in the diagrams are not relevant to the discussion in this work, and only the phase boundaries are here under appreciation. 
illumination $[21,48]$. However, even if fluid-fluid phase separation as seen by fluorescence microscopy were considered as non-reliable [24], two-component ESR spectra of the type shown in the lower part of Fig. 7 [171], the corresponding two-component ${ }^{2} \mathrm{H}-\mathrm{NMR}$ spectra in ternary systems in Veatch et al. [16], and the recent X-ray data from Chen et al. [143] and Mills et al. [144] provide consistent evidence for the existence of a distinct liquid-ordered phase.

One possible explanation for the failure of fluorescence microscopy to detect phase coexistence in Chol-containing binary mixtures is that $\mathrm{L}_{\mathrm{d}}$ and $\mathrm{L}_{\mathrm{o}}$ domains are simply too small to be resolved by optical microscopy, although other explanations involving formation of condensed lipid complexes have been offered [191,192]. These newer ideas describing condensed lipid complexes would provide a rationale for understanding ternary lipid mixtures containing cholesterol and help to unify data provided by monolayers and GUVs. Resolution of coexisting lipid phases by the spin-label ESR methods described here is not intrinsically dependent upon the domain size. Instead, what is required is that the differences in anisotropic rotational mobility or in the ordering of the lipids in the two phases should be large enough that they can be distinguished spectrally, and that lipid exchange between the domains should not be faster than the frequency equivalent of this spectral difference (see, for example, [171,193]). Residence times in the submicrosecond regime have been estimated for egg sphingomyelin-cholesterol mixtures, based on lifetime broadening of lipid spin-label spectra [141]. Results of this analysis led the authors to suggest the existence of molecular complexes that make up the $\mathrm{L}_{0}$-phase and coexist with disordered lipid. Such estimates lie at the sensitivity limit of conventional spinlabel ESR, and probably are similarly at an upper sensitivity limit for the exchange rates. There are also problems in reconciling this scenario with the phase rule (even considering that the sphingomyelin is a mixed species), and with the observation that the coexisting spin-label population in the mixed species system was more disordered than it was in SM alone in the $\mathrm{L}_{\alpha}$-phase. In spite of a number of examples of micrometer-sized cell membrane domains (see Section 3), the general trend appears to be in favour of the domains in the fluid coexistence regime being small.

\subsection{Model systems vs. cell membranes}

Although the lateral structure of some compositionally simple lipid systems has been extensively studied in the last 30 years, relatively little is known from studies in model systems about the effect of the compositional complexity of biological membranes, including membrane proteins, on the bilayer phase state. It is reasonable to suppose that the nature of the membrane proteins would have an effect, as would the membrane lipid/protein compositional ratio; in some cases biological membranes can contain up to $60-70 \%$ protein, so the protein presence would be likely to make a substantial contribution to the overall nature of the bilayer. In fact, previous studies have shown small but definite effects of intrinsic proteins on the gel-fluid transition [194-196], and on the lamellar-hexagonal transition [197]. Data extracted from lipid-only bilayer model systems have demonstrated that both the presence and the physical characteristics of phase coexistence are highly dependent on the lipid composition of the membrane itself and the particular environmental conditions such as the temperature. As a rule of thumb, particular lipid combinations among naturally occurring lipid species with high melting phase transition temperatures (i.e. those that are above physiological temperatures such as DPPC, SM, cerebrosides, ceramide), low melting phase transition temperatures (in general, unsaturated lipid species), and Chol may exhibit different phase coexistence scenarios. Some lipid mixtures such as DPPC or SM, with unsaturated phospholipids and Chol, may display $\mathrm{L}_{0}-\mathrm{L}_{\mathrm{d}}$ phase coexistence at certain temperatures and molar fractions, as proposed in the raft hypothesis [29,32,186]. This observation is important in providing some clues about the impact of these molecular species in the lateral structure of particular biological membranes. However, it is not obvious from the information reported in the literature whether the particular lipid composition of a given biological membrane should be first taken into account in order to predict the occurrence of lateral separation. The raft's lipid compositional information is often assumed to be general and not specific; this frequent oversight is particularly surprising. It is not uncommon to see many types of different cell membranes being tested for the presence of rafts, even though the lipid and protein compositional information (i.e. the different molecular species and their molar fraction) among these membranes may differ, and sometimes may differ substantially.

Phase diagrams of simple lipid mixtures together with visual information provided by microscopy techniques (fluorescence microscopy, atomic force microscopy) are useful tools for inferring the structural features of certain compositionally complex biological membranes. However, a crucial point here is to first define a proper model system in order to mimic the lateral structure of the target membrane. In this process, the presence of key lipid species and of some membrane proteins, as well as their respective molar fractions are important parameters to take into account; it is also useful to consider that a temporal dependence may also exist associated with the molar fraction profile. It is wise to be cautious with regard to generalizing information about the lateral structure of membranes, as the reverse often happens in discussions on the raft hypothesis. The lateral structure of biological membranes may be far from being a general phenomenon, and may occur only in a limited number of systems, or under a limited number of conditions. Biological membranes are adapted to different functions, as are their molecular compositions. Membrane lateral heterogeneity can be interpreted either as merely the result of compositional fluctuations that may be related to condensed complex formation among certain lipid species, or as a phase separation phenomenon. It is important to bear in mind that the presence or absence of these phenomena may depend on the particular characteristics of the target biological membrane.

For more than 25 years, lipids were erroneously considered to be randomly organized building blocks of biological membranes. We now have the opportunity to more accurately describe the role of lipids in the lateral structure of biological membranes and to consider their compositional heterogeneity. Considering the broad and often puzzling interpretations of the raft hypothesis, we must be careful not to similarly pigeonhole rafts as just another membrane building block.

\section{Acknowledgments}

F.M.G. and A.A. thank the Spanish Ministerio de Educación y Ciencia (grant No. BFU 2007-62062 and No. BFU 2008-01637) and the Basque Government (grant No. IT-461-07) for their support. Research in the laboratory of L.A.B. is funded by a grant from FNU and FSS, Denmark and by the Danish National Research Foundation (which supports the MEMPHYS-Center for Biomembrane Physics). R.E.B. gratefully acknowledges the continuing financial support provided by NIH/NIGMS GM45928 and by the Hormel Foundation. M.P. acknowledges support from F.C.T. (Portugal). J.T. thanks the Natural Sciences and Engineering Research Council of Canada.

\section{References}

[1] M.C. Phillips, B.D. Ladbrooke, D. Chapman, Molecular interactions in mixed lecithin systems, Biochim. Biophys. Acta 196 (1970) 35-44

[2] E.J. Shimshick, H.M. McConnell, Lateral phase separation in phospholipid membranes, Biochemistry 12 (1973) 2351-2360.

[3] C.W.M. Grant, S. Hong-Wei Wu, H.M. McConnell, Lateral phase separations in binary lipid mixtures: correlation between spin label and freeze-fracture electron microscopic studies, Biochim. Biophys. Acta 363 (1974) 151-158.

[4] B.R. Lentz, Y. Barenholz, T.E. Thompson, Fluorescence depolarization studies of phase transitions and fluidity in phospholipid bilayers. 2. Two-component phosphatidylcholine liposomes, Biochemistry 15 (1976) 4529-4537. 
[5] C.F. Schmidt, Y. Barenholz, T.E. Thompson, A nuclear magnetic resonance study of sphingomyelin in bilayer systems, Biochemistry 16 (1977) 2649-2655.

[6] C. Gebhardt, L. Gruler, E. Sackmann, On domain structure and local curvature in lipid bilayers and biological membranes, Z Naturforsch [C] 32 (1977) 581-596.

[7] M.K. Jain, H.B. White III, Long-range order in biomembranes, Adv. Lipid Res. 15 (1977) 1-60.

[8] T.E. Thompson, T.W. Tillack, Organization of glycosphingolipids in bilayers and plasma membranes of mammalian cells, Annu. Rev. Biophys. Biophys. Chem. 14 (1985) 361-386.

[9] T.E. Thompson, Y. Barenholz, R.E. Brown, M. Correa-Freire, W.W. Young, T.W. Tillack, Molecular organization of glycosphingolipids in phosphatidylcholine bilayers and biological membranes, in: L. Freysz, H. Dreyfus, R. Massarelli, S. Gatt (Eds.), Enzymes of Lipid Metabolism II, Plenum Press, NY, 1986, pp. 387-396.

[10] J.H. Ipsen, G. Karlstrom, O.G. Mouritsen, H. Wennerstrom, M.J. Zuckermann, Phase equilibria in the phosphatidylcholine-cholesterol system, Biochim. Biophys. Acta 905 (1987) 162-172.

[11] K. Simons, E. Ikonen, Functional rafts in cell membranes, Nature 387 (1997) 569-572.

[12] K. Simons, W.L. Vaz, Model systems, lipid rafts, and cell membranes, Annu. Rev. Biophys. Biomol. Struct. 33 (2004) 269-295.

[13] S.L. Veatch, S.L. Keller, Miscibility phase diagrams of giant vesicles containing sphingomyelin, Phys. Rev. Lett. 94 (2005) 148101-148104.

[14] C.M. McQuaw, L. Zheng, A.G. Ewing, N. Winograd, Localization of sphingomyelin in cholesterol domains by imaging mass spectrometry, Langmuir 23 (2007) 5645-5650.

[15] S. Karmakar, V.A. Raghunathan, S. Mayor, Phase behaviour of dipalmitoylphospatidylcholine (DPPC)-cholesterol membranes, J. Phys. Condens. Matter 17 (2005) S1177-S1182.

[16] S.L. Veatch, S.L. Keller, K. Gawrisch, Critical fluctuations in domain-forming lipid mixtures, Proc. Natl. Acad. Sci. U. S. A. 104 (2007) 17650-17655.

[17] N. Santos, M. Prieto, M.A. Castanho, Quantifying molecular partition into model systems of biomembranes: an emphasis on optical spectroscopic methods, Biochim. Biophys. Acta 1612 (2003) 123-135.

[18] R.F. de Almeida, J.W. Borst, A. Fedorov, M. Prieto, A.J. Visser, Complexity of lipid domains and rafts in giant unillamelar vesicles revealed by combining imaging and microscopic time-resolved fluorescence, Biophys. J. 93 (2007) 539-553.

[19] D. Lichtenberg, F.M. Goñi, H. Heerklotz, Detergent-resistant membranes should not be identified with membrane rafts, Trends Biochem. Sci. 30 (2005) 430-436

[20] M.L. Kraft, P.K. Weber, M.L. Longo, I.D. Hutcheon, S.G. Boxer, Chemical imaging of lipid domains by high-resolution secondary ion mass spectrometry, Science 313 (2006) 1948-1951.

[21] J. Zhao, J. Wu, H. Shao, F. Kong, N. Jain, G. Hunt, G. Feigenson, Phase studies of model biomembranes: macroscopic coexistence of Lalpha+Lbeta, with lightinduced coexistence of Lalpha+Lo Phases, Biochim. Biophys. Acta 1768 (11) (2007) 2777-2786.

[22] E. London, How principles of domain formation in model membranes may explain ambiguities concerning lipid raft formation in cells, Biochim. Biophys. Acta 1746 (2005) 203-220.

[23] J.R. Silvius, Partitioning of membrane molecules between raft and non-raft domains: insights from model-membrane studies, Biochim. Biophys. Acta 1746 (2005) 193-202.

[24] G.W. Feigenson, Phase boundaries and biological membranes, Annu. Rev. Biophys. Biomol. Struct. 36 (2007) 63-77.

[25] L. Loura, R. de Almeida, M. Prieto, Detection and characterization of membrane microheterogeneity by resonance energy transfer, J. Fluoresc. 11 (2001) 197-209.

[26] R.F. de Almeida, L.M.S. Loura, A. Fedorov, M. Prieto, Lipid rafts have different sizes depending on membrane composition: a time-resolved fluorescence resonance energy transfer study, J. Mol. Biol. 346 (2005) 1109-1120.

[27] M.L. Frazier, J.R. Wright, Pokorny, A. Fischer, P.F.F. Almeida, Investigation of domain formation in spingomyelin/cholesterol/POPC mixtures by fluorescence resonance energy transfer and Monte-Carlo simulations, Biophys. J. 92 (2007) 2422-2433.

[28] L. Loura, A. Fedorov, M. Prieto, Fluid-fluid membrane microheterogeneity. A fluorescence energy transfer study, Biophys. J. 80 (2001) 776-788.

[29] S.L. Veatch, S.L. Keller, Seeing spots: complex phase behavior in simple membranes, Biochim. Biophys. Acta 1746 (2005) 172-185.

[30] S.D. Connell, D.A. Smith, The atomic force microscope as a tool for studying phase separation in lipid membranes, Mol. Membr. Biol. 200623 (2006) 17-28.

[31] L.A. Bagatolli, To see or not to see: lateral organization of Biological membranes and fluorescence microscopy, Biochim. Biophys. Acta 1758 (2006) 1541-1556.

[32] N. Kahya, D. Scherfeld, K. Bacia, P. Schwille, Lipid domain formation and dynamics in giant unilamellar vesicles explored by fluorescence correlation spectroscopy, J. Struct. Biol. 147 (2004) 77-89.

[33] A. Celli, S. Beretta, E. Gratton, Phase fluctuations on the micron-submicron scale in GUVs composed of a binary lipid mixture, Biophys. J. 94 (2008) 104-116.

[34] A.C. Garner, D.A. Smith, N.M. Hooper, Visualization of detergent solubilization of membranes: implications for the isolation of rafts, Biophys. J. 94 (2008) $1326-1340$.

[35] T. Kaasgaard, C. Leidy, J.H. Crowe, O.G. Mouritsen, K. Jørgensen, Temperaturecontrolled structure and kinetics of ripple phases in one- and two-component supported lipid bilayers, Biophys. J. 85 (2003) 350-360.

[36] P.E. Milhiet, M.C. Giocondi, C. Le Grimellec, AFM imaging of lipid domains in model membranes, Sci. World J. 3 (2003) 59-74.
[37] H.A. Rinia, M.M.E. Snel, J.P.J.M. van der Eerden, B. de Kruijff, Visualizing detergent-resistant domains in model membranes with atomic force microscopy, FEBS Lett. 501 (2001) 92-96.

[38] J. Bernardino de la Serna, J. Perez-Gil, A.C. Simonsen, L.A. Bagatolli, Cholesterol rules: direct observation of the coexistence of two fluid phases in native pulmonary surfactant membranes at physiological temperatures, J. Biol. Chem. 279 (2004) 40715-40722.

[39] S. Chiantia, N. Kahya, P. Schwille, Raft domain reorganization driven by shortand long-chain ceramide: a combined AFM and FCS study, Langmuir 23 (14) (2007) 7659-7665

[40] M.H. Jensen, E.J. Morris, A.C. Simonsen, Domain shapes, coarsening, and random patterns in ternary membranes, Langmuir 23 (2007) 8135-8141.

[41] W.C. Lin, C.D. Blanchette, T.V. Ratto, M.L. Longo, Lipid asymmetry in DLPC/ DSPC-supported lipid bilayers: a combined AFM and fluorescence microscopy study, Biophys. J. 90 (2006) 228-237.

[42] W.-C. Lin, C.D. Blanchette, M.L. Longo, Fluid-phase chain unsaturation controlling domain microstructure and phase in ternary lipid bilayers containing GalCer and cholesterol, Biophys. J. 92 (2007) 2831-2841.

[43] A.C. Simonsen, L.A. Bagatolli, Structure of spin-coated lipid films and domain formation in supported membranes formed by hydration, Langmuir 20 (2004) 9720-9728.

[44] F. Tokumasu, A.J. Jin, G.W. Feigenson, J.A. Dvorak, Nanoscopic lipid domain dynamics revealed by atomic force microscopy, Biophys. J. 84 (2003) 2609-2618.

[45] L.A. Bagatolli, E. Gratton, Two photon fluorescence microscopy of coexisting lipid domains in giant unilamellar vesicles of binary phospholipid mixtures, Biophys. J. 78 (2000) 290-305.

[46] B.Y. Van Duyl, D. Gancher, V. Chupin, B. de Kruijff, J.A. Killian, Sphingomyelin is much more effective than saturated phpsphatidylcholine in excluding unsaturated phosphatidylcholine from domains formed with cholesterol, FEBS Lett. 547 (2003) 101-106.

[47] N. Kahya, D. Scherfeld, K. Bacia, B. Poolman, P. Schwille, Probing lipid mobility of raft-exhibiting model membranes by fluorescence correlation spectroscopy, J. Biol. Chem. 278 (2003) 28109-28115.

[48] A.G. Ayuyan, F.S. Cohen, Lipid peroxides promote large rafts: effects of excitation of probes in fluorescence microscopy and electrochemical reactions during vesicle formation, Biophys. J. 91 (2006) 2172-2183.

[49] T. Baumgart, G. Hunt, E.R. Farkas, W.W. Webb, G.W. Feigenson, Fluorescence probe partitioning between Lo/Ld phases in lipid membranes, Biochim. Biophys. Acta 1768 (2007) 2182-2194.

[50] M. Fidorra, S. Hartel, J. Ipsen, L.A. Bagatolli, Do giant unilamellar vesicles composed of binary lipid mixtures obey the lever rule?: A quantitative microscopy imaging approach, Biophys. J. 94 (2008) 1198 (abstract).

[51] D. Evanko, STEDy progress, Nat. Methods 3 (2006) 661.

[52] L.A. Bagatolli, Thermotropic behavior of lipid mixtures studied at the level of single vesicles: giant unilamellar vesicles and two-photon excitation fluorescence microscopy, Methods Enzymol. 367 (2003) 233-253.

[53] L.A. Bagatolli, E. Gratton, Two-photon fluorescence microscopy observation of shape changes at the phase transition in phospholipid giant unilamellar vesicles, Biophys. J. 77 (1999) 2090-2101.

[54] Deleted.

[55] J. Korlach, P. Schwille, W.W. Webb, G.W. Feigenson, Characterization of lipid bilayer phases by confocal microscopy and fluorescence correlation spectroscopy, Proc. Natl. Acad. Sci. U. S. A. 96 (1999) 8461-8466.

[56] C. Dietrich, L.A. Bagatolli, Z.N. Volovyk, N.L. Thompson, M. Levi, K. Jacobson, GrattonE. , Lipid rafts reconstituted in model membranes, Biophys. J. 80 (2001) 1417-1428.

[57] K. Nag, J.S. Pao, R.R. Harbottle, F. Possmayer, N.O. Petersen, L.A. Bagatolli, Segregation of saturated chain lipids in pulmonary surfactant films and bilayers, Biophys. J. 82 (2002) 2041-2051.

[58] M. Fidorra, L. Duelund, C. Leidy, A.C. Simonsen, L.A. Bagatolli, Absence of fluid-ordered/fluid-disordered phase coexistence in ceramide/POPC mixtures containing cholesterol, Biophys. J. 90 (2006) 4437-4451.

[59] I. Plasencia, L. Norlén, L.A. Bagatolli, Direct visualization of lipid domains in human skin stratum corneum's lipid membranes: effect of $\mathrm{pH}$ and temperature, Biophys. J. 93 (2007) 3142-3155.

[60] C. Dietrich, Z.N. Volovyk, M. Levi, N.L. Thompson, K. Jacobson, Partitioning of Thy-1, GM1, and cross-linked phospholipid analogs into lipid rafts reconstituted in supported model membrane monolayers, Proc. Natl. Acad. Sci. U.S.A. 98 (2001) 10642-10647

[61] K. Gaus, E. Gratton, E.P. Kable, A.S. Jones, I. Gelissen, L. Kritharides, W. Jessup, Visualizing lipid structure and raft domains in living cells with two-photon microscopy, Proc. Natl. Acad. Sci. U. S. A. 100 (2003) 15554-15559.

[62] K. Gousset, W.F. Wolkers, N.M. Tsvetkova, A.E. Oliver, C.L. Field, N.J. Walker, J.H. Crowe, F. Tablin, Evidence for a physiological role for membrane rafts in human platelets, J. Cell. Physiol. 190 (2002) 117-128.

[63] C. Leidy, K. Gousset, J. Ricker, W.F. Wolkers, N.M. Tsvetkova, F. Tablin, J.H. Crowe, Lipid phase behavior and stabilization of domains in membranes of platelets, Cell Biochem. Biophys. 40 (2004) 123-148.

[64] S.L. Veatch, I.V. Polozov, K. Gawrisch, S.L. Keller, Liquid domains in vesicles investigated by NMR and fluorescence microscopy, Biophys. J. 86 (2004) 2910-2922.

[65] Q. Ruan, M.A. Cheng, M. Levi, E. Gratton, W.W. Mantulin, Spatial-temporal studies of membrane dynamics: scanning fluorescence correlation spectroscopy (SFCS), Biophys. J. 87 (2004) 1260-1267.

[66] T. Baumgart, A.T. Hammond, P. Sengupta, S.T. Hess, D.A. Holowka, B.A. Baird, W.W. Webb, Large-scale fluid/fluid phase separation of proteins and lipids in 
giant plasma membrane vesicles, Proc. Natl. Acad. Sci. U. S. A. 104 (2007) 3165-3170.

[67] R. Montes, A. Alonso, F.M. Goñi, L.A. Bagatolli, Giant unilamellar vesicles electroformed from native membranes and organic lipid mixtures under physiological conditions, Biophys. J. 93 (2007) 3548-3554.

[68] E. Sackmann, A charge-decoration technique for studying the heterogeneity of coexistent monolayer phases by electron microscopy, Nature 313 (1985) 299-301.

[69] H.M. McConnell, Structures and transitions in lipid monolayers at the air-water interface, Annu. Rev. Phys. Chem. 42 (1991) 171-195.

[70] H.M. McConnell, M. Vrljic, Liquid-liquid immiscibility in membranes, Ann. Rev. Biophys. Biomol. Struct. 32 (2003) 469-492.

[71] R.E. Brown, H.L. Brockman, Using monomolecular films to characterize lipid lateral interactions, in: T.J. McIntosh (Ed.), Methods Molecular Biology, 398, Humana Press Inc., Totowa, NJ, 2007, pp. 41-58.

[72] J.M. Smaby, H.L. Brockman, R.E. Brown, Cholesterol's interfacial interactions with sphingomyelins and phosphatidylcholines: hydrocarbon chain structure determines the magnitude of condensation, Biochemistry 33 (1994) 9135-9142.

[73] J.M. Smaby, M. Momsen, V.S. Kulkarni, R.E. Brown, Cholesterol-induced interfacial area condensations of galactosylceramides and sphingomyelins with identical acyl chains, Biochemistry 35 (1996) 5696-5704.

[74] J.M. Smaby, M.M. Momsen, H.L. Brockman, E. Rhoderick, Brown, Phosphatidylcholine acyl unsaturation modulates the decrease in interfacial elasticity induced by cholesterol, Biophys. J. 73 (1997) 1492-1505.

[75] R.E. Brown, Sphingolipid organization in biomembranes: what physical studies of model membranes reveal, J. Cell Sci. 111 (1998) 1-9.

[76] X.-M. Li, M.M. Momsen, J.M. Smaby, H.L. Brockman, R.E. Brown, Cholesterol decreases the interfacial elasticity and detergent solubility of sphingomyelins, Biochemistry 40 (2001) 5954-5963.

[77] J.P. Slotte, Lateral domain formation in mixed monolayers containing cholesterol and dipalmitoylphosphatidylcholine or N-palmitoylsphingomyelin, Biochim. Biophys. Acta 1235 (1995) 419-427.

[78] S.L. Veatch, S.L. Keller, Organization in lipid membranes containing cholesterol, Phys. Rev. Lett. 89 (2002) 268101-268104.

[79] B.L. Stottrup, D.S. Stevens, S.L. Keller, Miscibility of ternary mixtures of phospholipids and cholesterol in monolayers and application to bilayer systems, Biophys. J. 88 (2005) 269-276.

[80] B.L. Stottrup, S.L. Keller, Phase behaviour of lipid monolayers containing DPPC and cholesterol analogs, Biophys. J. 90 (2006) 3176-3183.

[80bis] M.C. Phillips, The physical state of phospholipids and cholesterol in monolayers, bilayers, and membranes, Prog. Surf. Membr. Sci. 5 (1972) 139-221.

[81] D. Marsh, Lateral pressure in membranes, Biochim. Biophys. Acta 1286 (1996) 183-223.

[82] D. Marsh, Comment on interpretation of mechanochemical properties of lipid bilayer vesicles from the equation of state or pressure-area measurement of the monolayer at the air-water or oil-water interface, Langmuir 22 (2006) 2916-2919.

[83] S.L. Keller, W.H. Pitcher III, W.H. Huestis, H.M. McConnell, Red blood cells lipids form immiscible liquids, Phys. Rev. Lett. 81 (1998) 5019-5022.

[84] C. Yuan, L.J. Johnston, Atomic force microscopy studies of ganglioside GM1 domains in phosphatidylcholine and phosphatidylcholine/cholesterol bilayers, Biophys. J. 81 (2001) 1059-1069.

[85] C. Yuan, J. Furlong, P. Burgos, L.J. Johnston, The size of lipid rafts: an atomic force microscopy study of ganglioside GM1 domains in sphingomyelin/DOPC/cholesterol membranes, Biophys. J. 82 (2002) 2526-2535.

[86] J.C. Lawrence, D.E. Saslowsky, J.M. Edwardson, R.M. Henderson, Real-time analysis of the effects of cholesterol on lipid raft behavior using atomic force microscopy, Biophys. J. 84 (2003) 1827-1832.

[87] A.G. Sostarecz, C.M. McQuaw, A.G. Ewing, N. Winograd, Phosphatidylethanolamineinduced cholesterol domains chemically identified with mass spectrometric imaging, J. Am. Chem. Soc. 126 (2004) 13882-13883.

[88] C.M. McQuaw, A.G. Sostarecz, L. Zheng, A.G. Ewing, N. Winograd, Lateral heterogeneity of dipalmitoylphosphatidylethanolamine-cholesterol Langmuir-Blodgett films investigated with imaging time-of-flight secondary ion mass spectrometry and atomic force microscopy, Langmuir 21 (2005) 807-813.

[89] L. Zheng, C.M. McQuaw, A.G. Ewing, N. Winograd, Sphingomyelin/-phosphatidylcholine and cholesterol interactions studied by imaging mass spectrometry, J. Am. Chem. Soc. 129 (2007) 15730-15731.

[90] I.A. Boldyrev, X. Zhai, M.M. Momsen, H.L. Brockman, R.E. Brown, J.G. Molotkovsky, New BODIPY lipid probes for fluorescence studies of membranes, J. Lipid Res. 48 (2007) 1518-1532.

[91] J. Sot, M.I. Collado, J.L.R. Arrondo, A. Alonso, F.M. Goñi, Triton X-100-resistant bilayers: effect of lipid composition and relevance to the raft phenomenon, Langmuir 18 (2002) 2828-2835.

[92] J. Yu, D.A. Fischman, T.L. Steck, Selective solubilization of proteins and phospholipids from red blood cell membranes by nonionic detergents, J. Supramol. Struct. 1 (1973) 233-248.

[93] J.I. Gurtubay, F.M. Goni, J.C. Gomez-Fernandez, J.J. Otamendi, J.M. Macarulla, Triton $\mathrm{X}-100$ solubilization of mitochondrial inner and outer membranes, J. Bioenerg. Biomembranes 12 (1980) 47-70.

[94] N.M. Hooper, A.J. Turner, Ectoenzymes of the kidney microvillar membrane. Differential solubilization by detergents can predict a glycosyl-phosphatidylinositol membrane anchor, Biochem. J. 250 (1988) 865-869.

[95] D.A. Brown, J.K. Rose, Sorting of GPI-anchored proteins to glycolipid-enriched membrane subdomains during transport to the apical cell surface, Cell 68 (1992) 533-544.
[96] E.A. Dennis, Formation and characterization of mixed micelles of the nonionic surfactant Triton X-100 with egg, dipalmitoyl, and dimyristoyl phosphatidylcholines, Arch. Biochem. Biophys. 165 (1974) 764-773.

[97] M.A. Partearroyo, M.A. Urbaneja, F.M. Goni, Effective detergent/lipid ratios in the solubilization of phosphatidylcholine vesicles by Triton X-100, FEBS Lett. 302 (1992) 138-140.

[97bis] D. Lichtenberg, Characterization of the solubilization of lipid bilayers by surfactants, Biochim. Biophys. Acta. 821 (1985) 470-478.

[98] F.M. Goni, A. Alonso, Spectroscopic techniques in the study of membrane solubilization, reconstitution and permeabilization by detergents, Biochim. Biophys. Acta 1508 (2000) 51-68.

[99] S.K. Patra, A. Alonso, F.M. Goni, Detergent solubilization of phospholipid bilayers in the gel state: the role of polar and hydrophobic forces, Biochim. Biophys. Acta 1373 (1998) 112-118.

[100] H. Ahyayauch, B. Larijani, A. Alonso, F.M. Goñi, Detergent solubilisation of phosphatidylcholine bilayers in the fluid state: influence of the acyl chain structure, Biochim. Biophys. Acta 1758 (2006) 190-196.

[101] S.K. Patra, A. Alonso, J.L.R. Arrondo, F.M. Goñi, Liposomes containing sphingomyelin and cholesterol: detergent solubilisation and infrared spectroscopic studies, J. Liposome Res. 9 (1999) 247-260.

[102] C. Arnulphi, J. Sot, M. Garcia-Pacios, J.L. Arrondo, A. Alonso, F.M. Goñi, Triton X100 partitioning into sphingomyelin bilayers at subsolubilizing detergen concentrations: effect of lipid phase and a comparison with dipalmitoylphosphatidylcholine, Biophys. J. 93 (2007) 3504-3514.

[103] R. Hertz, Y. Barenholz, Permeability and integrity properties of lecithinsphingomyelin liposomes, Chem. Phys. Lipids 15 (1975) 138-156.

[104] M.B. Sankaram, T.E. Thompson, Interaction of cholesterol with various glycerophospholipids and sphingomyelin, Biochemistry 29 (1990) 10670-10675.

[105] A. Sáez-Cirión, A. Alonso, F.M. Goñi, T.P.W. McMullen, R.N. McElhaney, E.A. Rivas, Equilibrium and kinetic studies of the solubilisation of phospholipid-cholesterol bilayers by C12E8. The influence of lipid phase structure, Langmuir 16 (2000) 1960-1968

[106] Y. Tamba, T. Tanaka, T. Yahagi, Y. Yamashita, M. Yamazaki, Stability of giant unilamellar vesicles and large unilamellar vesicles of liquid-ordered phase membranes in the presence of Triton X-100, Biochim. Biophys. Acta 1667 (2004) 1-6.

[107] X. Xu, R. Bittman, G. Duportail, D. Heissler, C. Vilcheze, E. London, Effect of the structure of natural sterols and sphingolipids on the formation of ordered sphingolipid/sterol domains (rafts). Comparison of cholesterol to plant, fungal, and disease-associated sterols and comparison of sphingomyelin, cerebrosides, and ceramide, J. Biol. Chem. 276 (2001) 33540-33546.

[108] X.-M. Li, M.M. Momsen, H.L. Brockman, R.E. Brown, Sterol structure and sphingomyelin acyl chain length modulate lateral packing elasticity and detergent solubility in model membranes, Biophys. J. 85 (2003) 3788-3801.

[109] M.A. Urbaneja, J.L. Nieva, F.M. Goni, A. Alonso, The influence of membrane composition on the solubilizing effects of Triton X-100, Biochim. Biophys. Acta 904 (1987) 337-345.

[110] X. Xu, E. London, The effect of sterol structure on membrane lipid domains reveals how cholesterol can induce lipid domain formation, Biochemistry 39 (2000) 843-849.

[111] J. Wang, Megha, E. London, Relationship between sterol/steroid structure and participation in ordered lipid domains (lipid rafts): implications for lipid raft structure and function, Biochemistry 43 (2004) 1010-1018.

[112] J.T. Davies, E.K. Rideal, Interfacial Phenomena, 2nd ed., Academic Press, New York, 1963, p. 265

[113] S.R. Shaikh, V. Cherezov, M. Caffrey, W. Stillwell, S.R. Wassall, Interaction of cholesterol with a docosahexaenoic acid-containing phosphatidylethanolamine: trigger for microdomain/raft formation? Biochemistry 42 (2003) 12028-12037.

[114] S.R. Shaikh, A.C. Dumaual, A. Castillo, D. LoCascio, R.A. Siddiqui, W. Stillwell, S.R. Wassall, Oleic and docosahexaenoic acid differentially phase separate from lipid raft molecules: a comparative NMR, DSC, AFM, and detergent extraction study, Biophys. J. 87 (2004) 1752-1766.

[115] H. Heerklotz, Triton promotes domain formation in lipid raft mixtures, Biophys. J. 83 (2002) 2693-2701.

[116] H. Heerklotz, H. Szadkowska, T. Anderson, J. Seelig, The sensitivity of lipid domains to small perturbations demonstrated by the effect of Triton, J. Mol Biol. 329 (2003) 793-799.

[117] S. Keller, A. Tsamaloukas, H. Heerklotz, A quantitative model describing the selective solubilization of membrane domains, J. Am. Chem. Soc. 127 (2005) 11469-11476.

[118] S.H. Untrach, G.G. Shipley, Molecular interactions between lecithin and sphingomyelin. Temperature- and composition-dependent phase separation, J. Biol. Chem. 252 (1977) 4449-4457.

[119] W.I. Calhoun, G.G. Shipley, Sphingomyelin-lecithin bilayers and their interaction with cholesterol, Biochemistry 18 (1979) 1717-1722.

[120] Y. Barenholz, T.E. Thompson, Sphingomyelins in bilayers and biological membranes, Biochim. Biophys. Acta 604 (1980) 129-158.

[121] M.B. Ruiz-Arguello, M.P. Veiga, J.L. Arrondo, F.M. Goni, A. Alonso, Sphingomyelinase cleavage of sphingomyelin in pure and mixed lipid membranes. Influence of the physical state of the sphingolipid, Chem. Phys. Lipids 114 (2002) 11-20.

[122] A. Pokorny, L.E. Yandek, A.I. Elegbede, A. Hinderliter, P.F.F. Almeida, Temperature and composition dependence of the interaction of $\delta$-lysin with ternary mixtures of sphingomylelin/cholesterol/POPC, Biophys. J. 91 (2006) 2184-2197.

[123] B. Térová, J.P. Slotte, T.K. Nyholm, Miscibility of acyl-chain defined phosphatidylcholines with N-palmitoyl sphingomyelin in bilayer membranes, Biochim. Biophys. Acta 1667 (2004) 182-189. 
[124] D.A. Mannock, T.J. McIntosh, X. Jiang, D.F. Covey, R.N. McElhaney, Effects of natural and enantiomeric cholesterol on the thermotropic phase behavior and structure of egg sphingomyelin bilayer membranes, Biophys. J. 84 (2003) 1038-1046.

[125] T.K. Nyholm, M. Nylund, J.P. Slotte, A calorimetric study of binary mixtures of dihydrosphingomyelin and sterols, sphingomyelin, or phosphatidylcholine, Biophys. J. 84 (2003) 3138-3146.

[126] T.N. Estep, D.B. Mountcastle, Y. Barenholz, R.L. Biltonen, T.E. Thompson, Thermal behavior of synthetic sphingomyelin-cholesterol dispersions, Biochemistry 18 (1979) 2112-2117.

[127] F.X. Contreras, J. Sot, M.B. Ruiz-Arguello, A. Alonso, F.M. Goni, Cholesterol modulation of sphingomyelinase activity at physiological temperatures, Chem. Phys. Lipids 130 (2004) 127-134.

[128] R.M. Epand, Cholesterol in bilayers of sphingomyelin or dihydrosphingomyelin at concentrations found in ocular lens membranes, Biophys. J. 84 (2003) 3102-3110.

[129] R. Krivanek, L. Okoro, R. Winter, Effect of cholesterol and ergosterol on the compressibility and volume fluctuations of phospholipid-sterol bilayers in the critical point region: a molecular acoustic and calorimetric study, Biophys. J. 94 (2008) 3538-3548.

[130] M. Vist, J.H. Davis, Phase equilibria of cholesterol/dipalmitoylphosphatidylcholine mixtures: ${ }^{2} \mathrm{H}$ nuclear magnetic resonance and differential scanning calorimetry, Biochemistry 29 (1990) 451-464

[131] H. Heerklotz, A. Tsamaloukas, Gradual change or phase transition: characterizing fluid lipid-cholesterol membranes on the basis of thermal volume changes, Biophys. J. 91 (2006) 600-607.

[132] H. McConnell, A. Radhakrishnan, Theory of the deuterium NMR of sterolphospholipid membranes, Proc. Natl. Acad. Sci. U. S. A. 103 (2006) 1184-1189.

[133] S.A. Pandit, G. Khelashvili, E. Jakobsson, A. Grama, H.L. Scott, Latera organization in lipid-cholesterol mixed bilayers, Biophys. J. 92 (2007) 440-447.

[134] T.P. McMullen, R.N. McElhaney, New aspects of the interaction of cholesterol with dipalmitoylphosphatidylcholine bilayers as revealed by high-sensitivity differential scanning calorimetry, Biochim. Biophys. Acta 1234 (1995) 90-98.

[135] S.P. Soni, D.S. LoCascio, Y. Liu, J.A. Williams, R. Bittman, W. Stillwell, S.R. Wassall Docosahexaenoic acid enhances segregation of lipids between raft and nonraft domains: ${ }^{2} \mathrm{H}-\mathrm{NMR}$ study, Biophys. J. 95 (2008) 203-214.

[136] M. Gandhavadi, D. Allende, A. Vidal, S.A. Simon, T.J. McIntosh, Structure, composition, and peptide binding properties of detergent soluble bilayers and detergent resistant rafts, Biophys. J. 82 (2002) 1469-1482.

[137] R.M. Epand, Cholesterol in bilayers of sphingomyelin or dihydrosphingomyelin at concentrations found in ocular lens membranes, Biophys. J. 84 (2003) 3102-3110.

[138] V. Luzzati, in: D. Chapman (Ed.), Biological Membranes: Physical fact and function, Academic Press, London, 1968, pp. 71-123.

[139] P.R. Maulik, D. Atkinson, G.G. Shipley, X-ray scattering of vesicles of N-acyl sphingomyelins. Determination of bilayer thickness, Biophys. J. 50 (1986) 1071-1077.

[140] S.L. Veatch, S.L. Keller, Separation of liquid phases in giant vesicles of ternary mixtures of phospholipids and cholesterol, Biophys. J. 85 (2003) 3074-3083.

[141] C. Chachaty, D. Rainteau, C. Tessier, P.J. Quinn, C. Wolf, Building up of the liquidordered phase formed by sphingomyelin and cholesterol, Biophys. J. 88 (2005) 4032-4044.

[142] J.A. Clarke, A.J. Heron, J.M. Seddon, R.V. Law, The diversity of the liquid ordered (Lo) phase of phosphatidylcholine/cholesterol membranes: a variable temperature multinuclear solid-state NMR and X-ray diffraction study, Biophys. J. 90 (2006) 2383-2393

[143] L. Chen, Z. Yu, P.J. Quinn, The partition of cholesterol between ordered and fluid bilayers of phosphatidylcholine: a synchrotron X-ray diffraction study, Biochim. Biophys. Acta 1768 (2007) 2873-2881.

[144] T.T. Mills, S. Tristram-Nagle, F.A. Heberle, N.F. Morales, J. Zhao, J. Wu, G.E.S. Toombes, J.F. Nagle, G.W. Feigenson, Liquid-liquid domains in bilayers detected by wide angle X-ray scattering, Biophys. J. 95 (2008) 682-690.

[145] Y.K. Levine, M.H. Wilkins, Structure of oriented lipid bilayers, Nat. New. Biol. 230 (1971) 69-72.

[146] J. Pan, T.T. Mills, S. Tristram-Nagle, J.F. Nagle, Cholesterol perturbs lipid bilayers nonuniversally, Phys. Rev. Lett. 100 (2008) 198103.

[147] H.A. Scheidt, D. Huster, K. Gawrisch, Diffusion of cholesterol and its precursors in lipid membranes studied by $1 \mathrm{H}$ pulsed field gradient magic angle spinning NMR, Biophys. J. 89 (2005) 2504-2512.

[148] J. Pencer, T. Mills, V. Anghel, S. Krueger, R.M. Epand, J. Katsaras, Detection of submicron-sized raft-like domains in membranes by small-angle neutron scattering, Eur. Phys. J. E. Soft Matter 18 (2005) 447-458.

[149] J. Pencer, T. Mills, N. Kucerka, M.P. Nieh, J. Katsaras, Small-angle neutron scattering to detect rafts and lipid domains, Methods Mol. Biol. 398 (2007) 231-244.

[150] S.L. Veatch, S.S.W. Leung, R.E.W. Hancock, J.L. Thewalt, Fluorescent probes alter miscibility phase boundaries in ternary vesicles, J. Chem. Phys. B. 111 (2007) 502-504.

[151] Y.-W. Hsueh, M. Zuckermann, J. Thewalt, Phase diagram determination of phospholipid/sterol membranes using deuterium NMR, Concepts Magn. Reson. 26 A (2005) 35-46.

[152] G. Orädd, G. Lindblom, Lateral diffusion studied by pulsed field gradient NMR on oriented lipid membranes, Magn. Reson. Chem. 42 (2004) 123-131.

[153] A. Filippov, G. Orädd, G. Lindblom, The effect of cholesterol on the lateral diffusion of phospholipids in oriented bilayers, Biophys. J. 84 (2003) 3079-3086.

[154] F. Aussenac, M. Tavares, E.J. DuFourc, Cholesterol dynamics in membranes of raft composition: a molecular point of view from ${ }^{2} \mathrm{H}$ and ${ }^{31} \mathrm{P}$ solid-state NMR, Biochemistry 42 (2003) 1383-1390.
[155] A. Bunge, P. Müller, M. Stöckl, A. Herrmann, D. Huster, Characterization of the ternary mixture of sphingomyelin, POPC, and cholesterol. Support for an inhomogeneous lipid distribution at high temperatures, Biophys. J. 94 (2008) 2680-2690.

[156] Y.-W. Hsueh, R. Giles, N. Kitson, J. Thewalt, The effect of ceramide on phosphatidylcholine membranes: a deuterium NMR study, Biophys. J. 82 (2002) 3089-3095.

[157] A. Seelig, J. Seelig, Effect of a single cis double-bond on structure of a phospholipid bilayer, Biochemistry 16 (1977) 45-50.

[158] E. Oldfield, M. Meadows, D. Rice, R. Jacobs, Spectroscopic studies of specifically deuterium labeled membrane systems. Nuclear magnetic resonance investigation of the effects of cholesterol in model systems, Biochemistry 17 (1978) 2727-2740.

[159] D.E. Warschawski, P.F. Devaux, Order parameters of unsaturated phospholipids in membranes and the effect of cholesterol: $\mathrm{a}{ }^{1} \mathrm{H}-{ }^{13} \mathrm{C}$ solid-state NMR study at natural abundance, Eur. Biophys. J. 34 (2005) 987-996.

[160] W. Guo, V. Kurze, T. Huber, N.H. Afdahl, K. Beyer, J.A. Hamilton, A solid-state NMR study of phospholipid-cholesterol interactions: sphingomyelin-cholesterol binary systems, Biophys. J. 83 (2002) 1465-1478.

[161] R.M. Epand, R.F. Epand, Non-raft forming sphingomyelin-cholesterol mixtures, Chem. Phys. Lipids 132 (2004) 37-46.

[162] A.L. Costello, T.M. Alam, Using ${ }^{31} \mathrm{P}$ MAS NMR to monitor a gel phase thermal disorder transition in sphingomyelin/cholesterol bilayers, Biochim. Biophys. Acta 1778 (2008) 97-104.

[163] A. Filippov, G. Orädd, G. Lindblom, Lipid lateral diffusion in ordered and disordered phases in raft mixtures, Biophys. J. 86 (2004) 891-896.

[164] M. Lafleur, P.R. Cullis, M. Bloom, Modulation of the orientational order profile of the lipid acyl chain in the L-alpha phase, Eur. Biophys. J. 19 (1990) 55-62.

[165] J.L. Thewalt, M. Bloom, Phosphatidylcholine:cholesterol phase diagrams, Biophys. J. 63 (1992) 1176-1183.

[166] Y.-W. Hsueh, M.T. Chen, The liquid phase properties of POPC/cholesterol membranes: a deuterium NMR study, Biophys. J. (Suppl. S) (2007) 580A.

[167] D.E. Warschawski, P.F. Devaux, ${ }^{1} \mathrm{H}-{ }^{13} \mathrm{C}$ polarization transfer in membranes: a tool for probing lipid dynamics and the effect of cholesterol, J. Magn. Reson. 177 (2004) 166-171.

168] G. Orädd, P.W. Westerman, G. Lindblom, Lateral diffusion coefficients of separate lipid species in a ternary raft-forming bilayer: a Pfg-NMR multinuclear study, Biophys. J. 89 (2005) 315-320.

[169] I.V. Polozov, K. Gawrisch, Characterization of the liquid-ordered state by proton MAS NMR, Biophys. J. 90 (2006) 2051-2061.

[170] S.L. Veatch, K. Gawrisch, S.L. Keller, Closed-loop miscibility gap and quantitative tie-lines in ternary membranes containing diphytanoyl PC, Biophys. J. 90 (2006) 4428-4436.

[171] M.I. Collado, F.M. Goñi, A. Alonso, D. Marsh, Domain formation in sphingomyelin/cholesterol mixed membranes studied by spin-label electron spin resonance spectroscopy, Biochemistry 44 (2005) 4911-4918.

[172] Y.W. Chiang, Y. Shimoyama, G.W. Feigenson, J.H. Freed, Dynamic molecular structure of DPPC-DLPC-cholesterol ternary lipid system by spin-label electron spin resonance, Biophys. J. 87 (2004) 2483-2496.

173] Y.W. Chiang J. Zhao, J. Wu, Y.H. Shimoyama, J.H. Freed, G.W. Feigenson, New method for determining tie-lines in coexisting membrane phases using spinlabel ESR, Biochim. Biophys. Acta 1668 (2005) 99-105.

[174] M.P. Veiga, J.L.R. Arrondo, F.M. Goñi, A. Alonso, D. Marsh, Interaction of cholesterol with sphingomyelin in mixed membranes containing phosphatidylcholine, studied by spin-label ESR and IR spectroscopies. A possible stabilization of gel-phase sphingolipid domains by cholesterol, Biochemistry 40 (2001) 2614-2622.

[175] M.P. Veiga, F.M. Goñi, A. Alonso, D. Marsh, Mixed membranes of sphingolipids and glycerolipids studied by spin-label ESR spectroscopy. A search for domain formation, Biochemistry 39 (2000) 9876-9883.

[176] J.L.R. Rubenstein, J.C. Owicki, H.M. McConnell, Dynamic properties of binary mixtures of phosphatidylcholines and cholesterol, Biochemistry 19 (1980) 569-573.

[177] D.J. Recktenwald, H.M. McConnell, Phase equilibria in binary mixtures of phosphatidylcholine and cholesterol, Biochemistry 20 (1981) 4505-4510.

[178] M.B. Sankaram, T.E. Thompson, Cholesterol-induced fluid-phase immiscibility in membranes, Proc. Nat. Acad. Sci. U. S. A. 88 (1991) 8686-8690.

[179] K.Y.C. Lee, J.F. Klingler, H.M. McConnell, Electric field-induced concentration gradients in lipid monolayers, Science 263 (1994) 655-658.

[180] S. Pautot, B.J. Frisken, D.A. Weitz, Engineering asymmetric vesicles, Proc. Natl. Acad. Sci. U. S. A. 100 (2003) 10718-10721.

[181] J.R. Silvius, Role of cholesterol in lipid raft formation: lessons from lipid model systems, Biochim. Biophys. Acta 1610 (2003) 174-183.

[182] R. Merkel, E. Sackmann, E. Evans, Molecular friction and epitactic coupling between monolayers in supported bilayers, J. Phys. (Paris) 50 (1989) 1535-1555.

[183] S. Mayor, M. Rao, Rafts: scale-dependent, active lipid organization at the cell surface, Traffic 5 (2004) 231-240.

[184] P.F. Devaux, R. Morris, Transmembrane asymmetry and lateral domains in biological membranes, Traffic 5 (2004) 241-246.

[185] M.D. Collins, S.L. Keller, Tuning lipid mixtures to induce or suppress domain formation across leaflets of unsupported asymmetric bilayers, Proc. Natl. Acad. Sci. U. S. A. 105 (2008) 124-128.

[186] R.F. de Almeida, A. Fedorov, M. Prieto, Sphingomyelin/phosphatidylcholine/ cholesterol phase diagram: boundaries and composition of lipid rafts, Biophys. J. 85 (2003) 2406-2416. 
[187] R. Elliot, I. Szleifer, M. Schick, Phase diagram of a ternary mixture of cholesterol and saturated and unsaturated lipids calculated from a microscopic model, Phys. Rev. Lett. 96 (2006) 98-101.

[188] C. Nicolini, J. Baranski, S. Schlummer, J. Palomo, M. Lumbierres-Burgues, M. Kahms, J. Kuhlmann, S. Sanchez, E. Gratton, H. Waldmann, R. Winter, Visualizing association of N-Ras in lipid microdomains: influence of domain structure and interfacial adsorption, J. Am. Chem. Soc. 128 (2006) 192-201.

[189] P.F.F. Almeida, A. Pokorny, A. Hinderliter, Thermodynamics of membrane domains, Biochim. Biophys. Acta 1720 (2005) 1-13.

[190] R.F. de Almeida, J. Borst, A. Fedorov, M. Prieto, A.J. Visser, Complexity of lipid domains and rafts in giant unilamellar vesicles revealed by combining imaging and microscopic and macroscopic time-resolved fluorescence, Biophys. J. 2007 93 (2) (2007) 539-553.

[191] H.M. McConnell, Complexes in ternary cholesterol-phospholipid mixtures, Biophys. J. 88 (2005) L23-L25.
[192] A. Radhakrishnan, H. McConnell, Condensed complexes in vesicles containing cholesterol and phospholipids, Proc. Natl. Acad. Sci. U. S. A. 102 (2005) 12662-12666.

[193] D. Marsh, L.I. Horváth, in: A.J. Hoff (Ed.), Advanced EPR. Applications in Biology and Biochemistry, Elsevier, Amsterdam, 1989, pp. 707-752.

[194] D. Chapman, J.C. Gomez-Fernandez, F.M. Goni, Intrinsic protein-lipid interactions. Physical and biochemical evidence, FEBS Lett. 98 (1979) 211-223.

[195] E. Freire, T. Markello, C. Rigell, P.W. Holloway, Calorimetric and fluorescence characterization of interactions between cytochrome $b 5$ and phosphatidylcholine bilayers, Biochemistry 22 (1983) 1675-1680.

[196] M.R. Morrow, J.H. Davis, F.J. Sharom, M.P. Lamb, Studies on the interaction of human erythrocyte band 3 with membrane lipids using deuterium nuclear magnetic resonance and differential scanning calorimetry, Biochim. Biophys. Acta 858 (1986) 13-20.

[197] J.A. Killian, B. de Kruijff, Thermodynamic, motional, and structural aspects of gramicidin-induced hexagonal $\mathrm{H}_{\mathrm{II}}$ phase formation in phosphatidylethanolamine, Biochemistry 24 (1985) 7881-7890. 